\title{
Wafer-scale transfer route for top-down III-nitride nanowire LED arrays based on the femtosecond laser lift-off technique
}

Nursidik Yulianto (1) ${ }^{1,2,3}$, Andam Deatama Refino ${ }^{1,2,4}$, Alina Syring ${ }^{1,2}$, Nurhalis Majid ${ }^{3,5}$, Shinta Mariana ${ }^{1,2}$, Patrick Schnell ${ }^{6}$, Ruri Agung Wahyuono ${ }^{7}$, Kuwat Triyana ${ }^{8}$, Florian Meierhofer ${ }^{1,2}$, Winfried Daum ${ }^{5}$, Fatwa F. Abdi (1) ${ }^{6}$, Tobias Voss ${ }^{1,2}$, Hutomo Suryo Wasisto (id ${ }^{1,2}$ and Andreas Waag (10),

\begin{abstract}
The integration of gallium nitride (GaN) nanowire light-emitting diodes (nanoLEDs) on flexible substrates offers opportunities for applications beyond rigid solid-state lighting (e.g., for wearable optoelectronics and bendable inorganic displays). Here, we report on a fast physical transfer route based on femtosecond laser lift-off (fs-LLO) to realize wafer-scale top-down GaN nanoLED arrays on unconventional platforms. Combined with photolithography and hybrid etching processes, we successfully transferred GaN blue nanoLEDs from a full two-inch sapphire substrate onto a flexible copper $(\mathrm{Cu})$ foil with a high nanowire density $\left(\sim 10^{7}\right.$ wires $\left./ \mathrm{cm}^{2}\right)$, transfer yield $(\sim 99.5 \%)$, and reproducibility. Various nanoanalytical measurements were conducted to evaluate the performance and limitations of the $f_{s}$-LLO technique as well as to gain insights into physical material properties such as strain relaxation and assess the maturity of the transfer process. This work could enable the easy recycling of native growth substrates and inspire the development of large-scale hybrid GaN nanowire optoelectronic devices by solely employing standard epitaxial LED wafers (i.e., customized LED wafers with additional embedded sacrificial materials and a complicated growth process are not required).
\end{abstract}

\section{Introduction}

Gallium nitride $(\mathrm{GaN})$ has been continuously used in the light-emitting diode (LED) industry for almost three decades since the introduction of commercial high-brightness GaN blue LEDs in 1993 by Nakamura et al. ${ }^{1-3}$. Their invention has propelled the previously less-considered $\mathrm{GaN}$ materials into mainstream optoelectronic research and development. Today, GaN is ubiquitously found in a wide range of device applications beyond solid-state lighting (e.g., electronics, displays, optical microscopy, visible light communications, fuel cells, optogenetics, and biochemical

\footnotetext{
Correspondence: Nursidik Yulianto (n.yulianto@tu-braunschweig.de) or Hutomo Suryo Wasisto (h.wasisto@tu-braunschweig.de)

${ }^{1}$ Institute of Semiconductor Technology (IHT), Technische Universität Braunschweig, Hans-Sommer-Straße 66, Braunschweig 38106, Germany ${ }^{2}$ Laboratory for Emerging Nanometrology (LENA), Technische Universität Braunschweig, Langer Kamp 6, Braunschweig 38106, Germany

Full list of author information is available at the end of the article
}

sensors $)^{3-9}$. It is fascinating to witness that the interest in GaN LEDs is now shifting from standard, square millimeter-sized high-power LEDs to micrometer-sized pixels $(10-50 \mu \mathrm{m})^{10,11}$. Owing to their high brightness, high transparency, long lifetime, low power consumption, and short response time, GaN microLEDs are an ideal candidate for display applications and have the potential to replace liquid crystal display (LCD) and organic LED (OLED) technology ${ }^{12,13}$.

Considering the processing complexity, cost, and performance of these devices, hybrid integration of microLEDs with a transistor backplane is more preferable than a monolithic fabrication of high-electron-mobility transistors and microLEDs on a single wafer. Thus, GaN microLEDs grown on sapphires are commonly flip-chip bonded onto silicon-based complementary metal oxide semiconductor (CMOS) drivers, in which a laser lift-off

\section{() The Author(s) 2021}

(c) (i) Open Access This article is licensed under a Creative Commons Attribution 4.0 International License, which permits use, sharing, adaptation, distribution and reproduction cc) in any medium or format, as long as you give appropriate credit to the original author(s) and the source, provide a link to the Creative Commons license, and indicate if changes were made. The images or other third party material in this article are included in the article's Creative Commons license, unless indicated otherwise in a credit line to the material. If material is not included in the article's Creative Commons license and your intended use is not permitted by statutory regulation or exceeds the permitted use, you will need to obtain permission directly from the copyright holder. To view a copy of this license, visit http://creativecommons.org/licenses/by/4.0/. 
(LLO) transfer process subsequently releases the native substrate $^{14,15}$. For state-of-the-art LLO-based transfer technology in the LED industry, various short-pulsed laser sources (e.g., excimer and Q-switched nanosecond lasers $(355 \mathrm{~nm}))$ have frequently been used, where delamination of the GaN layer from the sapphire occurs based on a direct photon absorption mechanism in the $n-\mathrm{GaN}$ part located at the $\mathrm{GaN} / \mathrm{sapphire}$ interface ${ }^{16}$. In fact, this technological concept is mature and generally used in LED fabrication.

The further shrinkage of GaN-based LEDs to submicron sizes $(<1 \mu \mathrm{m})$ has been demonstrated by forming them as core-shell, disc-in, and dot-in indium gallium nitride $(\mathrm{InGaN}) / \mathrm{GaN}$ nanowire LEDs (nanoLEDs) in a bottom-up approach; these devices are known as nanoLEDs $^{11,17-19}$. InGaN/GaN nanoLED arrays with tunable peak emission wavelengths in almost the entire visible spectrum (red, green, and blue-RGB) can be obtained on a single wafer solely by varying the wire diameters $(200-600 \mathrm{~nm})^{11}$. Furthermore, since the initial demonstrations on self-assembled vertical $\mathrm{GaN}$ nanowires in the late 1990s, continuous improvements have been mainly devoted to controlling the bottom-up fabrication of random and aligned nanowires by molecular beam epitaxy and metal-organic chemical vapor deposition ${ }^{20-22}$. However, bottom-up $\mathrm{GaN}$ nanowire fabrication has revealed several limitations (i.e., difficult-to-control concentrations of impurities, defects along the wire in the $c$-plane direction, and the formation of inhomogeneous structures). Thus, in most cases, these selectively grown GaN nanowires possess different geometries (i.e., heights, diameters, and shapes), although their growth substrates have been lithographically patterned ${ }^{23,24}$. In contrast, the epitaxial growth of planar GaN film LEDs has reached a high level of maturity. Starting from planar heterostructures, nanoLEDs can also be fabricated by a top-down method, which has lately become more popular, obviating the disadvantages of the bottom-up approach by simply etching the planar thin-film LED into disc-in nanoLEDs ${ }^{24-26}$.

One of the key processes in microdisplay fabrication is the wafer-scale transfer of the micro/nanoLED structures onto another suitable carrier, e.g., a CMOS silicon wafer or a flexible metal foil. Flexible metal foils are particularly useful for wearable optoelectronic devices such as flexible displays and optical smart sensors. Earlier studies have demonstrated the simple integration of core-shell InGaN/ GaN nanoLEDs into a flexible substrate by embedding the devices within elastomeric polydimethylsiloxane (PDMS) layer ${ }^{27-29}$. Although several hybrid flexible GaN LEDs emitting blue, green, and white light were successfully fabricated in those reports, their main building blocks (i.e., self-assembled nanoLEDs) possessed random positions, irregular heights, and discordant geometries. Moreover, their wire transfer was still performed manually using a scalpel on a small device area $(1 \mathrm{~cm} \times 1.5 \mathrm{~cm})$, leading to a low process reproducibility level ${ }^{27}$. Another conventional nanowire LED transfer method through a pick-and-place process could take several hours in preparation, especially, when electron beam lithography was used to form contacts, causing a limited number of transferred nanowires (low yield) and inefficient integration ${ }^{30}$. Utilizing selective photoelectrochemical (PEC) etching, GaN LED devices could also be separated from their growth substrates. Nonetheless, this method is very sensitive to the doping concentration and requires a customized LED wafer with an additional embedded sacrificial material (e.g., zinc oxide $^{31}$, chromium nitride ${ }^{32}$, boron nitride ${ }^{33}$, or $\mathrm{InGaN}^{34}$ ), which definitely induces higher costs and more difficulties in optimizing heteroepitaxial growth.

Herein, we employed a fast physical transfer technique based on ultrashort femtosecond laser lift-off $(f s$-LLO) to realize vertical GaN nanoLEDs on nonconventional substrates (see Fig. 1). In our previous reports, $f s$-LLO at a wavelength of $520 \mathrm{~nm}$ and a pulse width of $350 \mathrm{fs}$ was used to release GaN LED films with small area sizes $(0.5 \times$ $0.5 \mathrm{~mm}^{2}$ and $1 \times 1 \mathrm{~cm}^{2}$ ) from their original sapphire substrates $^{35,36}$. However, in those studies, the GaN LEDs were either not fully fabricated as a completely functional device in a cleanroom (i.e., $n$ - $\mathrm{GaN}$ opening was not performed and metal contact was not realized $)^{35}$ or processed into non-nanostructured LED devices ${ }^{36}$. Moreover, although thin-film LEDs could be attached to the $\mathrm{Cu}$ foil, their flexibility on a large scale was not demonstrated. Thus, in this work, we investigated the potency of $f s$-LLO to transfer wafer-scale GaN nanoLEDs embedded in SU-8 from a sapphire substrate onto a $\mathrm{Cu}$ foil, in which commercial GaN LED wafers were used during laser processing. The greatest advantage of the $f s$-LLO technique is its ability to transfer the semiconductor film with a higher bandgap than the photon energy from the excitation laser source. This method could be applied to separate AlGaN UV LEDs from their sapphire substrates, where multiphoton absorption from a femtosecond laser occurred during the transfer process ${ }^{36}$. Therefore, $f_{s}$-LLO is a versatile technique for creating thin-film UV-visible GaN LEDs. Meanwhile, choosing a femtosecond laser source at visible wavelengths (e.g., $520 \mathrm{~nm}$ ) offers precise optical alignment during sample preparation. Different laser sources with a longer wavelength of $1030 \mathrm{~nm}$ (nearinfrared) and a pulse width of $350 \mathrm{fs}$ can also be used to yield multiphoton absorption instead of only two-photon absorption. This technique has been reported to slice $\mathrm{GaN}$ thin film from its original bulk substrate (i.e., bulk $\mathrm{GaN})^{37}$.

Furthermore, highly ordered nanowires were fabricated in a top-down approach from full two-inch InGaN/GaN LED wafers combining photolithography, inductively coupled plasma reactive ion etching (ICP-RIE), and wet 


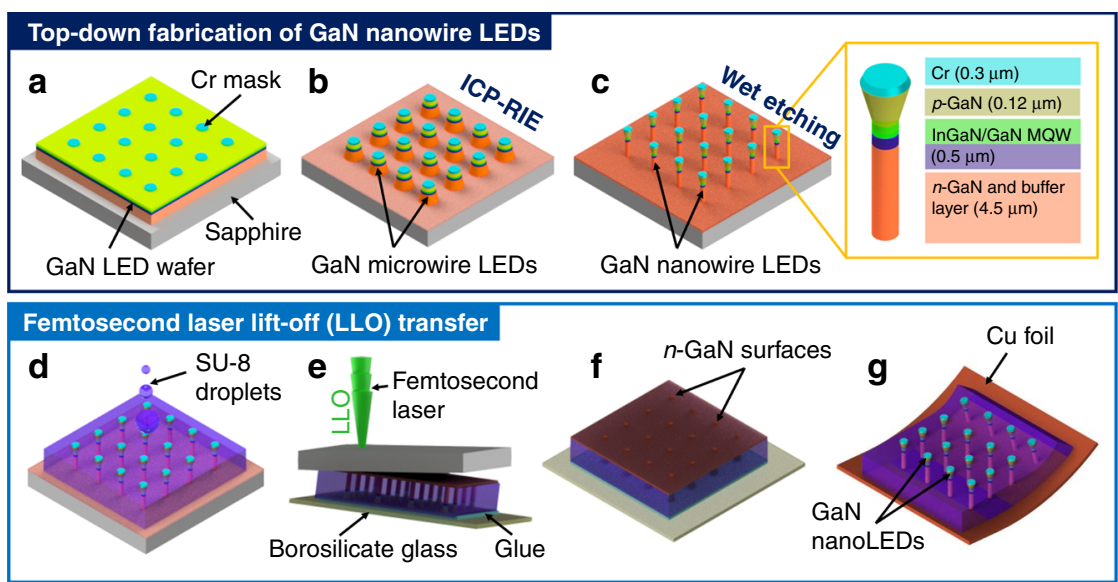

Fig. 1 Wafer-scale transfer route for vertical GaN nanowire light-emitting diodes (nanoLEDs) on a nonconventional substrate. a The top-down fabrication includes photolithography to pattern chromium (Cr) masks on an LED wafer, $\mathbf{b}$ inductively coupled plasma reactive ion etching (ICP-RIE) using an $\mathrm{SF}_{6} / \mathrm{H}_{2}$ gas mixture at room temperature, and $\mathbf{c}$ potassium hydroxide (KOH)-based wet etching. The femtosecond laser lift-off (fs-LLO) transfer process consists of $\mathbf{d}$ polymer spin-coating and back-etching, e transfer of the whole GaN nanoLED structure from sapphire onto temporary borosilicate glass substrate and its subsequent delamination from the sapphire using a femtosecond laser, $\mathbf{f}$ sapphire removal resulting in the opening of an $n$-GaN surface, and $\mathbf{g}$ cleaning of GaN nanoLEDs attached to a copper (Cu) foil with the freely exposed $p$-GaN layer on the top side

etching. SU-8 polymer and copper $(\mathrm{Cu})$ foil were used as flexible substrates to keep the nanowire arrays in good order and to act as bottom $n$-contacts, respectively. Compared with other flexible substrates made of polymer materials (e.g., polyethylene terephthalate, PDMS, and Kapton polyimide films) ${ }^{38}, \mathrm{Cu}$ foil provides higher electrical and thermal conductivities in addition to good flexibility $^{39}$. Moreover, the SU-8 thin layer is a transparent and flexible material that is adaptable to the bending of $\mathrm{Cu}$ foil substrates ${ }^{40,41}$. The thickness of SU-8 can be precisely adjusted using oxygen plasma-based dry etching. The material characteristics of the transferred nanoLEDs were investigated to assess the strength and weakness of the $f s$-LLO transfer process. The transferred $\mathrm{GaN}$ nanoLEDs address the aforementioned manufacturing challenges because they satisfy the small device dimension requirement, can be separated from their rigid native growth substrates straightforwardly, require the only standard LED wafers, and have a high potential to be employed for flexible display platforms, all achieved with a rapid, nonchemical, and scalable method.

\section{Results and discussion Top-down GaN nanoLEDs}

A hybrid etching method comprising sequential $\mathrm{SF}_{6} /$ $\mathrm{H}_{2}$-based ICP-RIE and $\mathrm{KOH}$-based wet etching processes was employed to form vertical GaN nanoLEDs out of $\sim 4.6 \mu \mathrm{m}$ thick planar GaN LED film grown on a sapphire substrate (Fig. 1a-c). During lithography, $300 \mathrm{~nm}$ thick spherical chromium $(\mathrm{Cr})$ islands with a diameter of $1 \mu \mathrm{m}$ were created from a $\mathrm{Cr}$ film that had been previously deposited by electron beam evaporation. A metal-based hard mask of the Cr layer was required instead of a photoresist or nanoimprint resist, which is commonly used in the top-down fabrication of vertical silicon (Si) nanowire arrays ${ }^{42-45}$. In terms of the mechanical properties, GaN has a higher hardness, mechanical stability, and stiffness (i.e., Young's modulus of 300-350 GPa) than $\mathrm{Si}^{46,47}$. Thus, the $\mathrm{Cr}$ mask must be sustained during the first deep physical etching of the GaN nanoLEDs down almost to the sapphire substrate surface with an etch depth of $\sim 4.6 \mu \mathrm{m}$. It was reported in an ICP-RIE study that a $\mathrm{Cr}$ mask had high selectivity (up to 8:1) towards $\mathrm{GaN}$, which led to the feasible realization of a microscale $\mathrm{GaN}$ waveguide on $\mathrm{Ga}_{2} \mathrm{O}_{3}{ }^{48}$. For the etching selectivity, its value strongly depends on the parameters used, in which for physical etching, as in our case, the main influencing parameters are ICP power, high frequency (HF) power, and plasma density ${ }^{49}$. On the one hand, to support their application as a bendable optoelectronic device, $\mathrm{GaN}$ nanoLEDs should be completely separated from each other. On the other hand, when further device transfer steps (Fig. $1 \mathrm{~d}-\mathrm{g}$ ) are considered, having a very thin $n$-GaN layer holding all the GaN nanoLEDs is more beneficial for maintaining good vertical alignment during processing. Thus, in our transfer route, depending on the employed ICP-RIE recipe, the GaN nanoLEDs are mechanically supported by a remaining $n$-GaN layer with a thickness of 200-400 nm after hybrid etching.

To precisely define the required etching duration during ICP-RIE, we first conducted experiments with dry etching times $\left(t_{\mathrm{ICP}}\right)$ varying from 10 to $50 \mathrm{~min}$ at $10 \mathrm{~min}$ intervals. The other etching parameters were kept constant (i.e., ICP power of $800 \mathrm{~W}$, HF power of $275 \mathrm{~W}$, DC bias of 


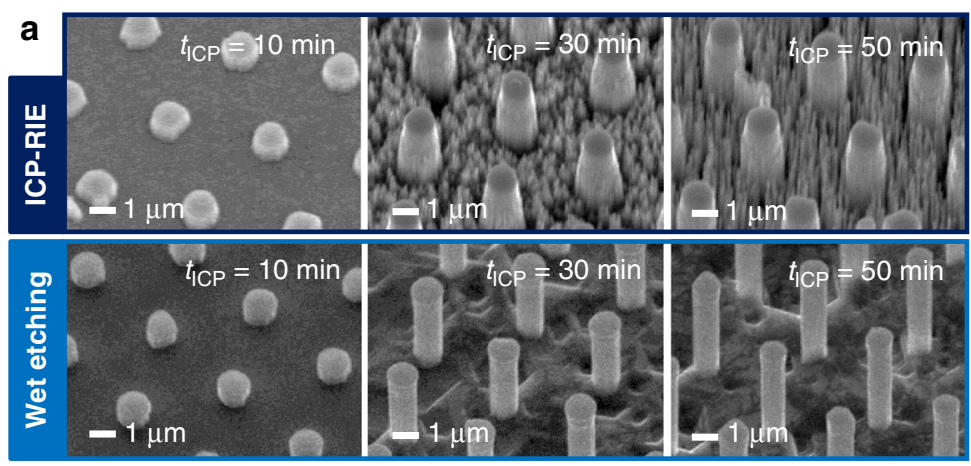

\section{b}
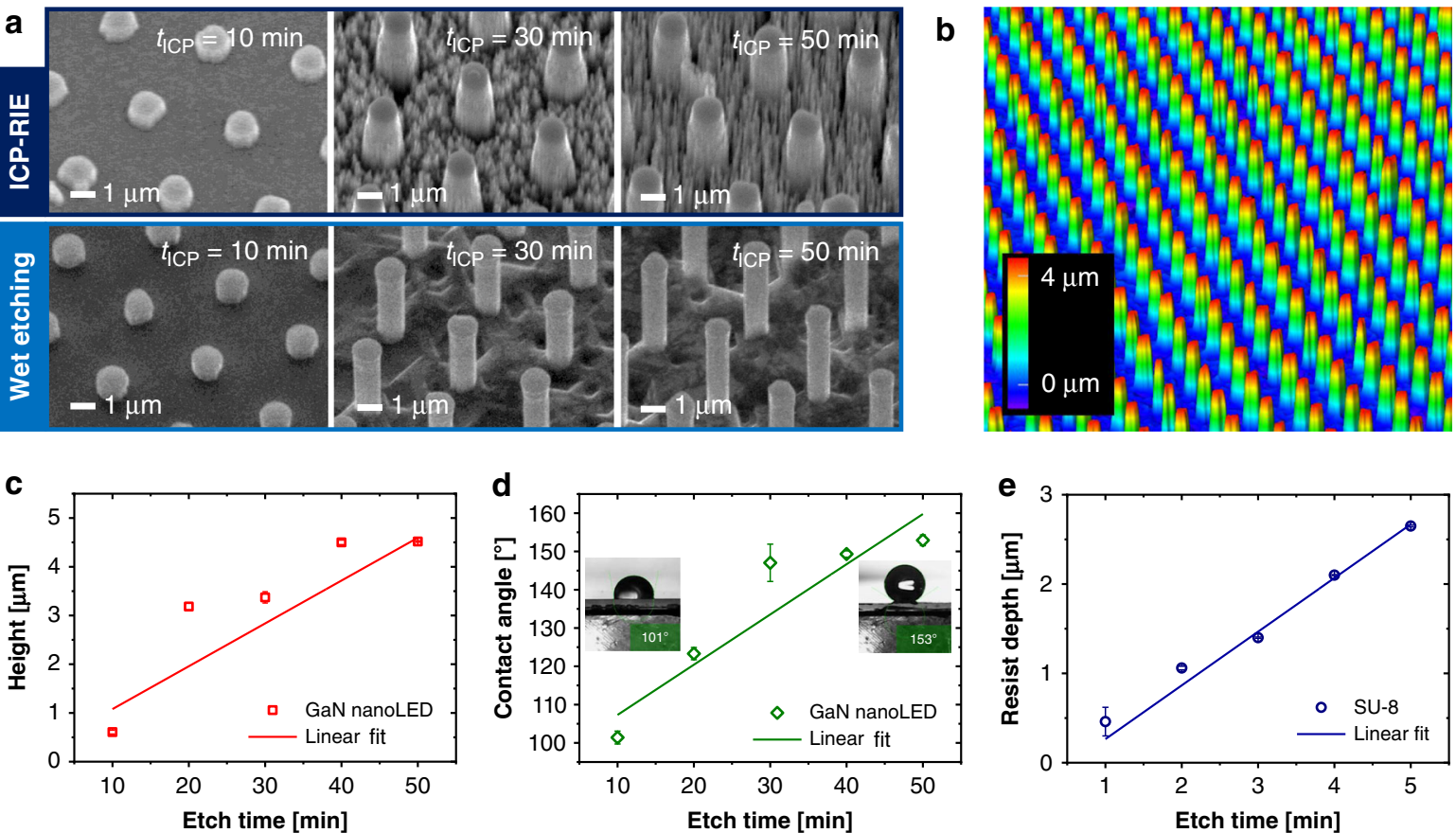

Fig. 2 GaN nanoLEDs created by a top-down hybrid etching method (i.e., a process combination of ICP-RIE and wet-chemical etching). a Scanning electron microscopy (SEM) images of vertical GaN nanoLEDs processed with different ICP-RIE times ( $t_{i C P}=10-50 \mathrm{~min}$ at room temperature) and constant wet etching durations $\left(20 \mathrm{~min}\right.$ at $\left.80^{\circ} \mathrm{C}\right)$. b 3D contour plot of typical GaN nanoLEDs ( $\left.t_{\mathrm{ICP}}=50 \mathrm{~min}\right)$ measured by confocal laser scanning microscopy (CLSM). c Influence of ICP-RIE time on nanoLED height. $\mathbf{d}$ Water contact angles measured on GaN nanoLEDs with different heights. e ICP-RIE duration against the etched SU-8 resist depth

$-140 \mathrm{~V}$, pressure of $0.5 \mathrm{~Pa}$, and $\mathrm{SF}_{6} / \mathrm{H}_{2}$ fluxes of $12 /$ $100 \mathrm{sccm}$ at room temperature). Fig. $2 \mathrm{a}$ depicts the scanning electron microscopy (SEM) images of the GaN nanoLEDs after ICP-RIE (top) and subsequent wetchemical etching processes (bottom). A longer dry etching duration not only results in higher nanowires but also leads to rougher surfaces that occur on the nanowire sidewalls and the bottom spaces among them. Nonetheless, these rough sidewall surfaces of the $\mathrm{GaN}$ nanowires could be smoothed by wet-chemical etching in a potassium hydroxide $(\mathrm{KOH})$-based etchant at a temperature of $80^{\circ} \mathrm{C}$ for $20 \mathrm{~min}$. Thus, the wire diameter also decreased. In case excessive etching time was used, flashlight- or mushroom-like GaN structures would appear instead of GaN cylinders because the wet etching proceeded at a slower rate in both the $p-\mathrm{GaN}$ and the multiquantum well $(\mathrm{MQW})$ region $^{50,51}$. Fig. $2 \mathrm{~b}$ shows a confocal laser scanning microscopy image of wafer-scale GaN nanoLED arrays with high homogeneity after dry and wet etching processes. Their typical diameter, height, and pitch are $\sim 700 \mathrm{~nm}, \sim 4.5 \mu \mathrm{m}$, and $4 \mu \mathrm{m}$, respectively.

Figure 2c indicates that for etch times longer than $40 \mathrm{~min}$, the dry etching process in the vertical direction slowed down. The fabricated GaN nanoLEDs have similar heights of $(4.50 \pm 0.05)$ and $(4.57 \pm 0.01) \mu \mathrm{m}$ when they were etched within 40 and $50 \mathrm{~min}$, respectively.
This phenomenon was caused by the higher level of grasslike structures appearing on the bottom $\mathrm{GaN}$ surface of the etched area, which prevented further etching (see Fig. 2a and S1a in the Supplementary Information). The occurrence process is very similar to the black silicon phenomenon, where an etching/passivation competition mechanism can lead to the creation of random $\mathrm{Si}$ nanospikes with very high aspect ratios in a self-masking way $^{52,53}$. When various carrier substrates (i.e., $\mathrm{Si}, \mathrm{Ti}, \mathrm{Al}$, and sapphire) were used underneath the GaN films, they could impact the surface profiles of the etched GaN films owing to unwanted contaminants during etching, resulting in different surface roughness levels ${ }^{54,55}$. In our case, running a longer etching process $(>50 \mathrm{~min})$ would therefore only further attack the $\mathrm{Cr}$ mask and lead to more dominant GaN grasses (Fig. S1a-e in the Supplementary Information). From these experiments, the dry etching rates for $\mathrm{GaN}$ and $\mathrm{Cr}$ were found to be 900 and $5 \mathrm{~nm} / \mathrm{min}$, respectively. In other words, a high selectivity of 180:1 was obtained for $\mathrm{Cr}$ as a mask for $\mathrm{GaN}$ etching. At $t_{\mathrm{ICP}}=70 \mathrm{~min}$, the $\mathrm{Cr}$ mask was completely stripped off from the GaN nanowire top surface during the ICP-RIE process. Thus, irregular $\mathrm{GaN}$ nanorods were yielded since the generated ions in the chamber could directly bombard the GaN top surfaces (Fig. S1c in the Supplementary Information). Afterwards, they were diminished when the 
sample was chemically etched in a $\mathrm{KOH}$-based solution (Fig. S1e in the Supplementary Information). This method was proven to depend on the target etch depth. A stable hard metal mask that is a few hundred nanometers has to be used during both dry and wet etching processes. The similar obtained nanowire heights for $t_{\mathrm{ICP}}=20$ and 30 min could be due to the variation in chamber condition during the etching process (i.e., the ICP etcher was normally used for structuring not only GaN but also other materials ( $\mathrm{Si}$ and $\mathrm{SU}-8$ resist)). In addition to being implemented for nanoLEDs, this hybrid technique has been previously employed for building $\mathrm{GaN}$ nanowire field-effect transistors with various layer stack compositions (e.g., $n-\mathrm{GaN}$ and $n-p-n-\mathrm{GaN})^{56-58}$.

In terms of the etching plasma used, despite their high toxicity, chlorine (Cl)- or boron (B)-based gases (e.g., $\mathrm{BCl}_{3} / \mathrm{Cl}_{2}, \mathrm{BCl}_{3} / \mathrm{SF}_{6}, \mathrm{~N}_{2} / \mathrm{Cl}_{2} / \mathrm{O}_{2}, \mathrm{Cl}_{2} / \mathrm{Ar} / \mathrm{O}_{2}, \mathrm{Cl}_{2} / \mathrm{N}_{2}$, and $\mathrm{Cl}_{2} / \mathrm{Ar}$ ) were still frequently used for etching $\mathrm{GaN}$ structures because of their high selectivity and verticality levels ${ }^{59-63}$. However, as in our case, other plasma sources (e.g., $\mathrm{SF}_{6} / \mathrm{H}_{2}$ ) can also be used for the dry etching of $\mathrm{GaN}$, although they do not deliver a very smooth surface on the sidewalls due to less chemical selectivity ${ }^{51,54,64}$. For LED devices, however, having smooth sidewalls is a prerequisite before the structures can be further processed. Rough sidewalls with numerous dangling bonds may constitute nonradiative recombination centers, which reduce the photon extraction efficiency ${ }^{26,65}$. Thus, $\mathrm{KOH}-$ based etching was carried out to overcome this issue.

Because of its non-centrosymmetric crystal structure, the wurtzite GaN crystal has a polar axis along the (0001) direction ${ }^{66}$. Although the N-polar direction possesses a structure with a single nitrogen bond oriented towards the surface, the Ga-polar direction comprises three nitrogen bonds oriented towards the surface. The N- and Ga-polar GaN materials demonstrated different etching behaviors when they were treated in $\mathrm{KOH}$ solution interacting with hydroxide ions $\left(\mathrm{OH}^{-}\right)^{67-69}$. To guarantee the $\mathrm{GaN}$ etching occurrence, the hydroxide ions need to have access to the Ga atom. Although hexagonal GaN pyramids can be formed in the N-polar region during etching, Ga-polar areas will remain unetched and perfectly intact ${ }^{67}$. During the initial GaN nanowire etching, $\mathrm{Ga}$ atoms are attacked and dissolved by $\left(\mathrm{OH}^{-}\right)$, forming gallium oxide $\left(\mathrm{Ga}_{2} \mathrm{O}_{3}\right)$ and ammonia $\left(\mathrm{NH}_{3}\right)$. The vertical nonpolar sidewalls have a high density of nitrogen dangling bonds, which are negatively charged and can electrostatically repel the hydroxide ions, thus protecting the gallium back bonds and controlling the etching process ${ }^{70}$. However, if numerous defects exist in the GaN material, such as those found in our microwires after ICP-RIE, a higher number of dangling or defective $\mathrm{Ga}$ bonds can promote local etch pit formation, which then allows the hydroxide ions to reach $\mathrm{Ga}$ atoms, progressing to the wet etching process. We note that the presence of a top $\mathrm{Cr}$ mask is a critical prerequisite to obtain smooth $a$-plane sidewalls. This was proven in our previous report, where $\mathrm{GaN}$ nanowires with free $c$-plane (without $\mathrm{Cr}$ mask) were wet etched in AZ400K, resulting in irregular cross-section profiles and $m$-plane sidewall ${ }^{51}$. Indeed, several factors may influence the wet etching rate (e.g., $\mathrm{KOH}$ concentration, temperature, etching time, mask material, and solution agitation). In our case, the lateral etching rate of $\mathrm{GaN}$ nanowires is typically in the range of $15-20 \mathrm{~nm} / \mathrm{min}^{26}$.

Although this work has focused on the investigation of the feasibility of the $f_{S}$-LLO-based transfer technique for large-scale $\mathrm{GaN}$ nanowire arrays, we additionally conducted wettability experiments on etched GaN nanoLEDs to predict their behavior when interacting with water droplets. This is critical if such platforms will be used as PEC sensors involving the photoluminescence (PL) detection technique ${ }^{71}$. For flexible GaN nanowire optoelectronic devices, silver $(\mathrm{Ag})$ nanowire networks or graphene materials that are diluted in water are often drop-cast or spincoated as transparent contact electrodes instead of thin indium tin oxide (ITO) films ${ }^{72-74}$. Fig. $2 \mathrm{~d}$ shows that by keeping the same pitch of $4 \mu \mathrm{m}$ and increasing the height of $\mathrm{GaN}$ nanowires from 1 to $4.6 \mu \mathrm{m}$, the measured water contact angle was changed from $101^{\circ}$ to $153^{\circ}$ (i.e., becoming more hydrophobic). Retaining hydrophobicity levels $\left(>150^{\circ}\right)$ that are too high will not be beneficial for dropping or spin-coating the water-based Ag nanowires, as the droplets will be strongly repelled by the $\mathrm{GaN}$ nanowires, leading to ineffective material contact deposition. However, a hydrophilic surface with a contact angle of $<90^{\circ}$ is also unwanted, as the deposited Ag nanowires will not be distributed homogenously over the wafer ${ }^{75}$. Thus, for optimal wettability conditions, a range between $90^{\circ}$ and $150^{\circ}$ is suggested.

In addition to wetting behavior, another important parameter is the etching rate of the SU-8 resist, which was used here as a mechanical support layer in addition to the remaining thin $n$-GaN film during the transfer process of the GaN nanoLEDs from the sapphire substrate to the $\mathrm{Cu}$ foil (Fig. 1d-g). This embedding polymer could also be used as an insulating layer to avoid a short circuit between $n$-GaN and $p$-GaN layers during $p$-contact creation ${ }^{27}$. The initial thickness of the spin-coated SU-8 was $\sim 5 \mu \mathrm{m}$, which completely covered the GaN nanoLEDs (Fig. S2a, b in the Supplementary Information). An etch rate of $600 \mathrm{~nm} / \mathrm{min}$ was found for the hardened SU-8 resist (see Fig. 2e) when it was etched in an ICP machine using $\mathrm{SF}_{6} /$ $\mathrm{O}_{2}$ plasma. This value is comparable with that in another SU-8 etching study employing the same plasma, in which etching rates of up to $800 \mathrm{~nm} / \mathrm{min}$ could be obtained with low roughness (i.e., a root mean square surface roughness of $<750 \mathrm{~nm}$ ) and high anisotropy ${ }^{76}$. Therefore, to reopen 


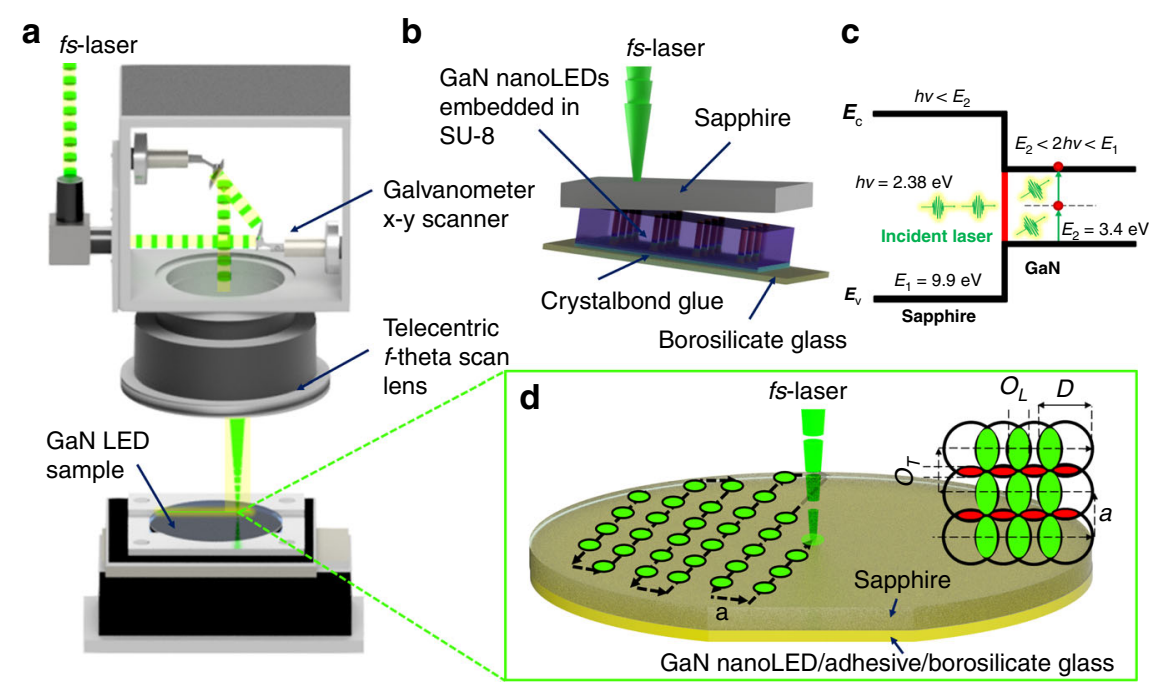

Fig. 3 Laser system configuration and micromachining pattern of $f s$-LLO for GaN nanoLEDs. a Laser beam path in the setup during the $f s$-LLO process based on raster scanning utilizing a Galvano $x$-y scanner. b Schematic of fs-LLO for GaN nanoLEDs embedded in an SU-8 layer. The laser light is transmitted through the wafer backside (sapphire). c Energy band diagram of $f_{s}$-LLO illustrating two-photon absorption in GaN material using femtosecond pulses at $520 \mathrm{~nm}$. The excitation of two photons ( $2 \mathrm{hv}$ ) from the laser possesses higher energy than GaN $\left(E_{2}\right)$ but lower energy than the sapphire $\left(E_{1}\right)$ bandgap. $\mathbf{d}$ Scheme of the $f_{S}$-LLO pattern showing pulse overlap and number. $O_{L}$ and $O_{T}$ are the percentage amounts of overlap between the diameters of two consecutive pulses in the lateral and transverse directions, respectively. $D$ and $a$ are assigned to diameter of craters in the $n$-GaN layer caused by the beam spot on the GaN surface and the track displacement (transverse pitch between passes), respectively

the top surface of the GaN nanoLEDs (Fig. 1e and S2c in the Supplementary Information), the filling SU-8 layer needs to be back-etched for 1-2 min depending on its original thickness.

\section{fs-LLO transfer}

After the GaN nanoLEDs had been prepared and filled with SU-8 resist, they needed to be temporarily bonded onto borosilicate glass as an intermediate carrier during the $f s$-LLO process. A thin Crystalbond glue layer heated at a temperature of $120^{\circ} \mathrm{C}$ was employed to realize the attachment forming the sapphire/GaN nanoLED/adhesive/borosilicate glass stack (Fig. 1e). This thermoplastic adhesive enables simple fast bonding and the removal of parts by thermal cycling. Thus, it has been widely used in the processing of several optoelectronic devices, including flexible optical neural probes embedded with GaN microLEDs ${ }^{77}$.

A laser source with a peak wavelength $(\lambda)$ of $520 \mathrm{~nm}$, a pulse width $(\tau)$ of $350 \mathrm{fs}$, and a repetition rate $(f)$ of $200 \mathrm{kHz}$ was used. The $f s$-LLO process was conducted by raster scanning of the femtosecond laser beam across the backside of the sapphire substrate (see Fig. 3a-d). Sapphire has a large energy bandgap $\left(E_{1}=9.9 \mathrm{eV}\right)$ and is transparent with respect to the laser wavelength. Therefore, photons emitted from the laser source are transmitted through the sapphire and subsequently reach the $\mathrm{GaN} /$ sapphire interface. Here, although the excitation laser photons possess lower energy $(h v=2.38 \mathrm{eV})$ than the $\mathrm{GaN}$ bandgap $\left(E_{2}=3.4 \mathrm{eV}\right)$, their high intensity leads to multiphoton absorption in the GaN region near the $\mathrm{GaN} /$ sapphire interface, as shown in Fig. 3c. This process is made possible by two-photon absorption ${ }^{35}$. To obtain a homogenous morphology while the laser beam hit the GaN surface, the $f s$-LLO procedure was performed with a laser scanning speed $(v)$ of $2 \mathrm{~m} / \mathrm{s}$, a scan pitch or distance between two pulses in the transverse direction $(a)$ of $10 \mu \mathrm{m}$, and a beam width $(D)$ of $\sim 20 \mu \mathrm{m}$ at the laser focus position on the target surface (see Fig. 3d). Thus, the working distance around the focal region is $\sim 0 \mathrm{~mm}$ from the surface of the sample ${ }^{35}$, whereas the distance between the sample surface and the telecentric lens is $\sim 12 \mathrm{~cm}$. Using Eq. (S3.1) and (S3.2) in the Supplementary Information, the degree of overlap between the diameters of two consecutive pulses in the lateral $\left(O_{L}\right)$ and transverse $\left(O_{T}\right)$ directions was determined to be $\sim 50 \%$. Moreover, the pulse number per area $(n / A)$ can be calculated using Eq. (S3.3), resulting in one pulse for every $100 \mu \mathrm{m}^{2}$. The laser pulse energy $\left(E_{p}\right)$ was kept constant at $\sim 3.3 \mu \mathrm{J}$ per pulse, corresponding to a laser energy density or peak fluence $\left(\Phi_{0}\right)$ of $\sim 1.5 \mathrm{~J} / \mathrm{cm}^{2}$ at the sample plane. In our previous report, this pulse energy level was proven to be able to yield a high lift-off success rate of up to $\sim 70 \%{ }^{35}$. The integrated fluence $\left(\Phi_{\text {int }}\right)$ calculated from Eq. (S3.4) amounts to $3.3 \mathrm{~J} / \mathrm{cm}^{2}$ (Fig. S3a, b in the Supplementary Information).

The conditions of the InGaN/GaN nanoLEDs in the entire process chain of the wafer-scale $f s$-LLO transfer 


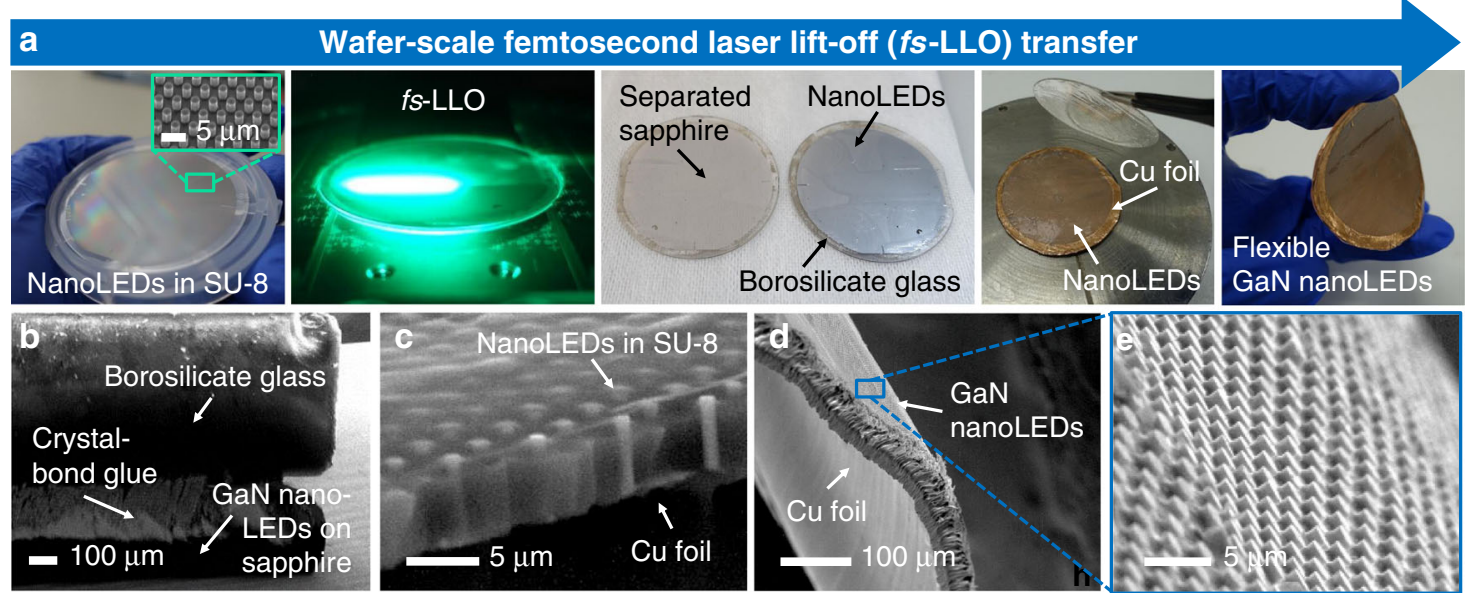

Fig. $4 \mathrm{GaN}$ nanoLEDs in the entire process chain of the wafer-scale fs-LLO transfer route starting from their top-down patterning to hybrid integration onto a Cu foil. a Photographs of the wafer-scale $f_{s}$-LLO transfer route for creating flexible GaN nanoLEDs: spin-coating of SU-8 on a nanopatterned LED wafer, etching of SU-8 by an $\mathrm{SF}_{6} / \mathrm{O}_{2}$-based ICP-RIE process exposing the $p$-GaN surface, fs-LLO processing, the release of nanoLEDs from a sapphire substrate, attachment of nanoLEDs onto Cu foil, and acetone cleaning (left-to-right). b GaN nanoLEDs on sapphire after being attached to a temporary borosilicate glass substrate. c Released GaN nanoLEDs after fs-LLO and attachment to Cu foil. The nanoLED arrays were embedded in an SU-8 membrane and still fully covered with Crystalbond glue. d-e GaN nanoLEDs that were embedded in a SU-8 membrane and attached to a flexible Cu foil after the cleaning procedure

route starting from their top-down patterning to hybrid integration onto a $\mathrm{Cu}$ foil are presented in Fig. 4a and Video S1 in the Supplementary Material. Prior to $f s$-LLO, the top-down patterned GaN nanoLEDs were filled with SU-8 resist followed by a hardening process. Then, they were tied to a temporary transfer substrate of $500 \mu \mathrm{m}$ thick borosilicate glass using Crystalbond adhesive at a temperature of $120^{\circ} \mathrm{C}$, in which a vertical force of $1 \mathrm{~N}$ was applied during the bonding procedure (Fig. 4b). By multiphoton absorption during the $f s$-LLO process, GaN was decomposed into metallic $\mathrm{Ga}$ and gaseous $\mathrm{N}_{2}$ over the whole wafer area. The presence of residual metallic $\mathrm{Ga}$ covering the $n-\mathrm{GaN}$ surface of the released sample was apparent from the metallic reflectivity ${ }^{78}$. Although $\mathrm{N}_{2}$ was released from the sample to the air, the oxidation of $\mathrm{Ga}$ in ambient $\mathrm{O}_{2}$ resulted in $\mathrm{Ga}_{2} \mathrm{O}_{3}$ layer on the surface ${ }^{79}$. To remove the $\mathrm{Ga}$ debris and $\mathrm{Ga}_{2} \mathrm{O}_{3}$ layer, the sample was cleaned using a mixed solution of (1:1) hydrochloric acid $(\mathrm{HCl})$ and deionized water $\left(\mathrm{DI} \mathrm{H}_{2} \mathrm{O}\right)$ for $2 \mathrm{~min}$ at room temperature $^{80}$. This cleaning process can be accelerated using boiled $\mathrm{HCl}^{81,82}$. The lifted-off $\mathrm{GaN}$ nanoLEDs were then attached to a $\mathrm{Cu}$ foil. As a result, the stacking of borosilicate glass/Crystalbond glue/GaN nanoLEDs on the SU-8/Cu film could be realized. Removal of the borosilicate glass was performed at a temperature of $120^{\circ} \mathrm{C}$ when the crystalbond adhesive was simultaneously melted. To completely strip off the adhesive residues, the integrated LED stack was cleaned with acetone and isopropanol. Once the temporary borosilicate glass substrate had been pulled away from the LED chips, a wafer-scale
GaN nanoLED arrays on $\mathrm{Cu}$ foil could be yielded (Fig. 4c-e). It should be noted that for further $p$-contact creation, an ICP-RIE process using $\mathrm{SF}_{6} / \mathrm{O}_{2}$ gases for 1-2 min was required. Hence, the top parts of $\mathrm{GaN}$ nanowires that were previously embedded in the SU-8 membrane could be freely exposed (Fig. 4e).

To estimate the transfer success rate of this $f s$-LLO for GaN nanoLEDs, the surface conditions of a full two-inch released sapphire wafer were investigated after release using an optical microscope (see Fig. S4a-e in the Supplementary Information). We employed the image analysis program Image to calculate the areas containing defects (nonlifted GaN nanoLEDs). The transfer failures were identified from the dark areas in the captured images. Although the homogeneity of optoelectrical characteristics of the GaN nanoLEDs was not tested in this case because the $p$-contact over the whole wafer surfaces had not been created and optimized (i.e., the investigation was limited to the physical appearance of the transferred nanowires on the borosilicate glass and $\mathrm{Cu}$ foil, as depicted by Fig. S4f, $g$ in the Supplementary Information), a transfer success rate of $\sim 99.5 \%$ could be obtained with a density of $2.5 \times 10^{7}$ wires $/ \mathrm{cm}^{2}$. In addition, SEM images were also taken at several different positions from the wafer-scale $\mathrm{GaN}$ nanoLEDs on $\mathrm{Cu}$ foil (see Fig. S4h-k in the Supplementary Information). Their large homogenous distribution over the carrier substrate can be confirmed by means of the captured nanoLED arrays located at the edge and middle areas. Small cracks of the SU- 8 and $n-\mathrm{GaN}$ supporting layers were also found after device bending. 


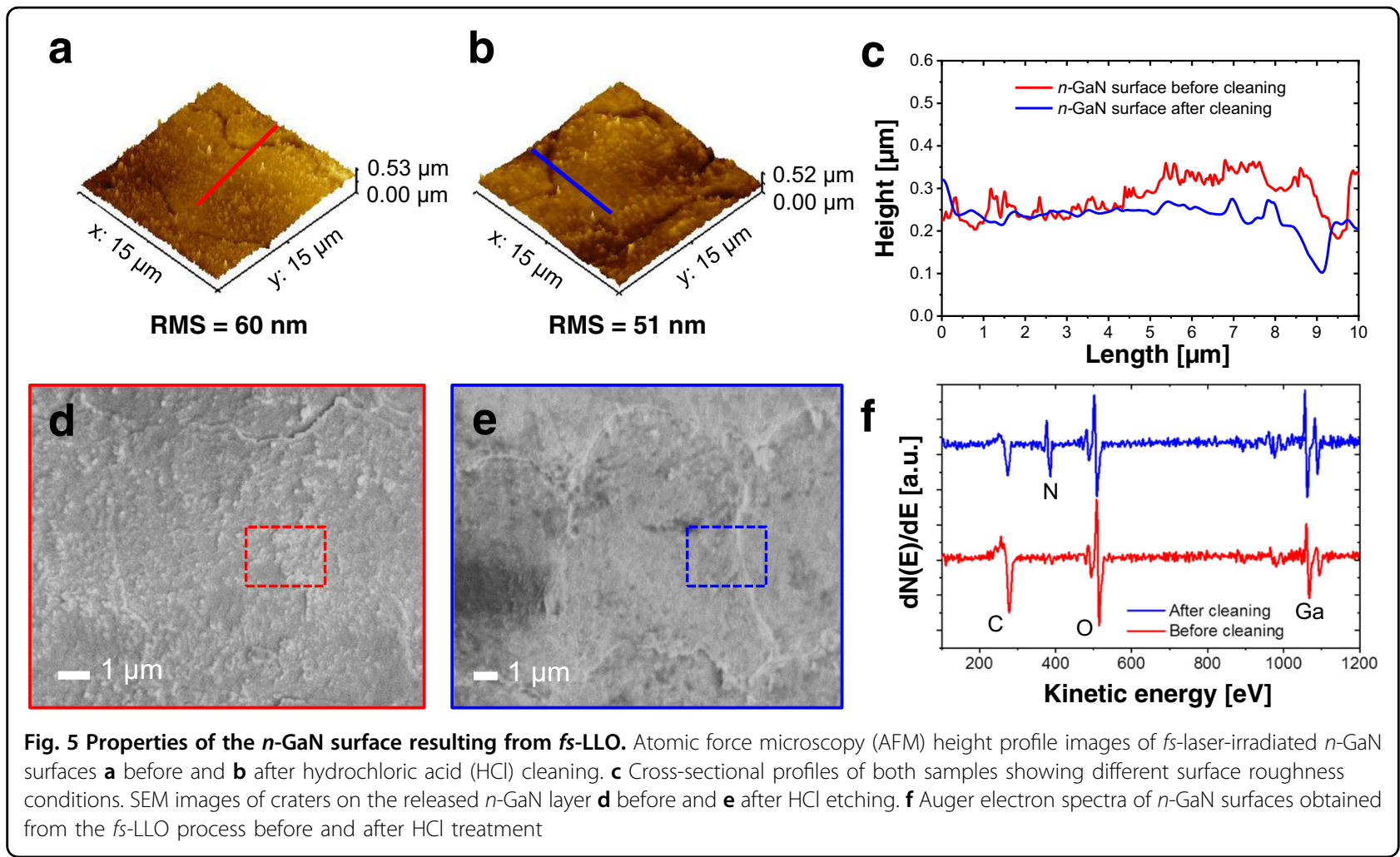

\section{NanoLED material characteristics}

Several methods for material characterization were applied to obtain insights into the relevant processes involved in the GaN nanoLED transfer route. First, we studied the effect of $\mathrm{HCl}$-based cleaning on the $\mathrm{GaN}$ surface morphology and composition after $f s$-LLO using atomic force microscopy (AFM) and Auger electron spectroscopy (AES). This chemical treatment can be employed to remove part of the Ga debris on the surface of the detached $n$-GaN layer, which may hinder the formation of good contact for the final device ${ }^{81,82}$ From the AFM measurements, root mean square roughness values of 60 and $51 \mathrm{~nm}$ were determined for a laser-irradiated, detached $n$-GaN surface before and after $\mathrm{HCl}$ cleaning, respectively (see Fig. $5 \mathrm{a}-\mathrm{c}$ ). This shows that this chemical etching could assist in the formation of smoother GaN surfaces. This argument is also supported by the SEM images showing a large part of a GaN crater (see Fig. 5d) and the overlapping region between two adjacent craters (see Fig. 5e), indicating that protrusions on the surfaces were diminished after the sample had been dipped in a diluted $\mathrm{HCl}$ solution. Nonetheless, the pattern of the laser beam with a scan pitch of $10 \mu \mathrm{m}$ could still be observed, with maximum crater depths of $\sim 15$ and $\sim 10 \mathrm{~nm}$ before and after $\mathrm{HCl}$ etching, respectively.

In addition to their topography, the released $n$-GaN surfaces were characterized by AES to analyze their elemental composition (see Fig. 5f). Prior to $\mathrm{HCl}$ cleaning, the surface region of the as-lifted sample contained $\sim 31.9 \% \mathrm{O}$ and $44.5 \% \mathrm{C}$, and the Ga-to-N concentration ratio was 6.4. Applying $\mathrm{HCl}$ etching reduced both the $\mathrm{O}$ and $\mathrm{C}$ contents to $26.5 \%$ and $25.4 \%$, respectively. The Gato- $\mathrm{N}$ ratio dropped to a value of 1.7 , suggesting that in addition to most of the carbon contamination, the $\mathrm{Ga}$ debris on the surface was largely etched away. The residual oxygen $\mathrm{AES}$ signal originates at least partly from the native surface oxidation of $\mathrm{GaN}$ to $\mathrm{Ga}_{2} \mathrm{O}_{3}$ in the air after etching. From another study related to the fabrication of 3D microchannels in bulk $\mathrm{GaN}$ by wet-chemical-assisted $f s$-laser ablation, it was experimentally demonstrated using X-ray photoelectron spectroscopy (XPS) that the chemical composition of the GaN surfaces was altered when they were cleaned using $\mathrm{HCl}$ solution ${ }^{78}$. The Ga-to$\mathrm{N}$ concentration ratios were reduced from 1.82 to 1.67 when the $f s$-laser-irradiated $\mathrm{GaN}$ surfaces had been treated in the $\mathrm{HCl}$ etching process. This reduced value was attributed to the removal of a Ga-rich layer or debris on the ablated surfaces. In addition to $\mathrm{HCl}$ solution, other chemicals (e.g., ammonium hydroxide $\left(\mathrm{NH}_{4} \mathrm{OH}\right)$, ammonium sulfide $\left(\left(\mathrm{NH}_{4}\right)_{2} \mathrm{~S}\right)$, hydrofluoric acid (HF), $\mathrm{KOH}$, and buffered oxide etch solutions) have been applied to remove contaminants, including native surface oxides on GaN surfaces. In these studies, AFM, AES, and XPS have been commonly utilized to compare the results among different cleaning techniques ${ }^{83-85}$. Solutions of $\mathrm{HCl}, \mathrm{HF}$, and $\mathrm{NH}_{4} \mathrm{OH}$ were found to be effective for oxide 


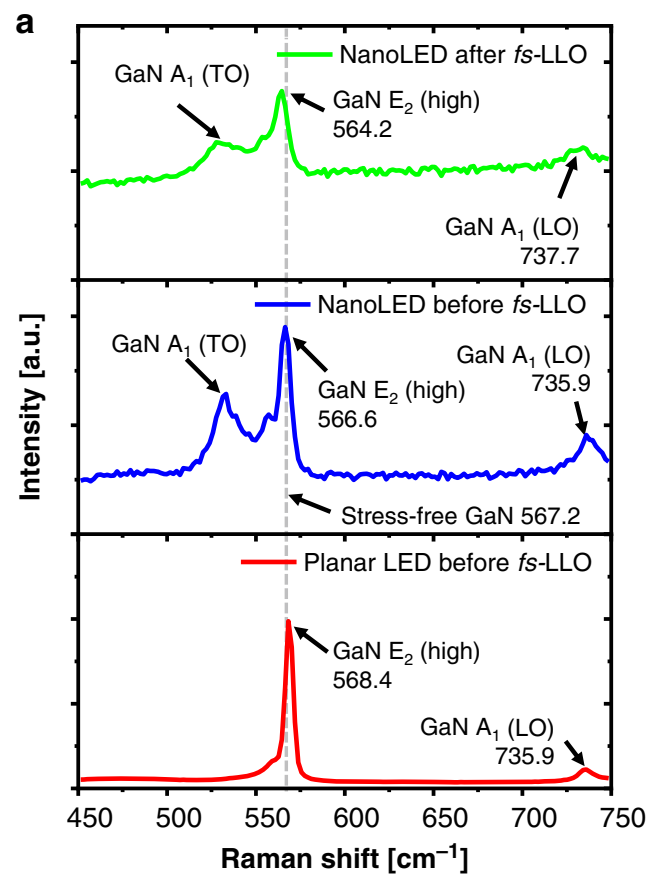

d

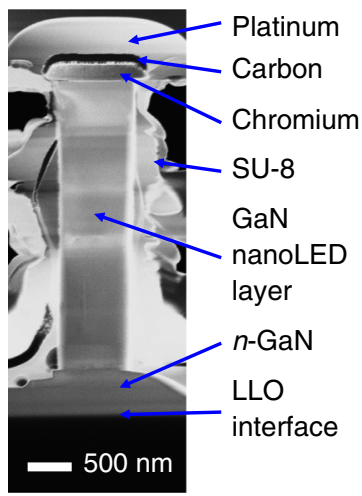

e
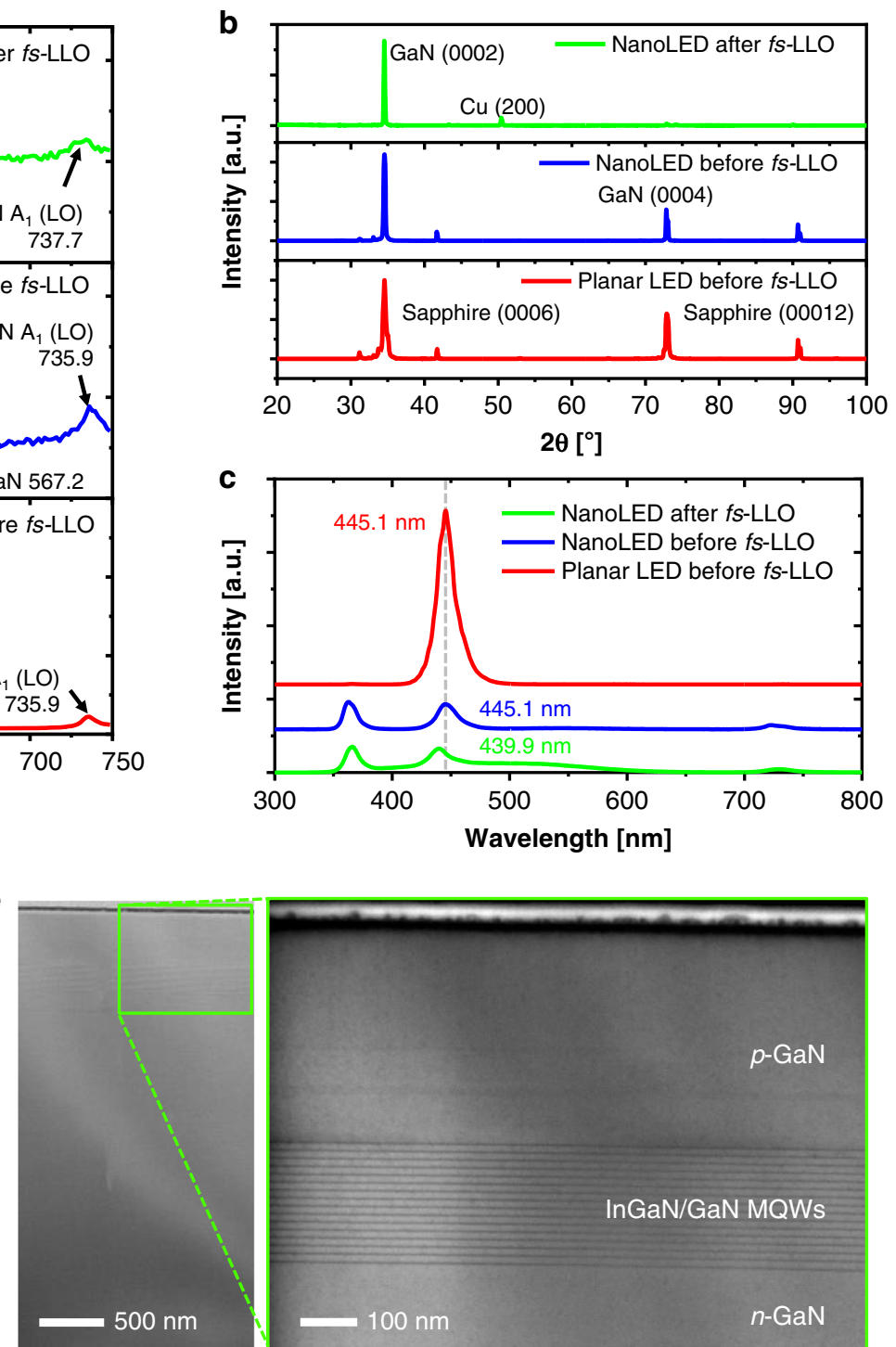

Fig. 6 Material characteristics of GaN planar LED and nanoLEDs before and after fs-LLO. a Raman, b X-ray diffraction (XRD), and c photoluminescence spectra of the InGaN/GaN nanoLEDs before and after fs-LLO in comparison with those of the InGaN/GaN planar LED. All measurements were carried out at room temperature. Cross-sectional focus-ion beam (FIB)-SEM images of $\mathbf{d}$ single GaN nanoLEDs and e thinfilm LEDs released by fs-LLO. A thin platinum-on-carbon layer was deposited on the LED top surface during sample preparation. The 16-fold InGaN/GaN multiquantum wells (MQWs) were still intact after laser irradiation

removal $^{80}$. It was also suggested that the cleaning process can be more effective when annealing or heating is involved. Using thermal desorption at a temperature of $>900^{\circ} \mathrm{C}$, effective removal of all contaminant species (e.g., carbon contaminants) on the $\mathrm{GaN}$ surface was demonstrated for $\mathrm{HCl}$-treated samples ${ }^{80,83}$.

As both nanostructuring and $f s$-LLO processing could potentially affect the crystalline and optical properties of the initially metal-organic vapor-phase epitaxy (MOVPE)grown LED stack, further analysis of three different samples (i.e., planar LED and nanoLEDs before and after $f_{S}$-LLO) at different stages of the wafer processing route was carried out. Figure 6a compares the Raman spectra of the pristine planar LED with those of the nanoLED before and after $f s$-LLO. All samples were examined in the backscattering direction with the excitation laser beam perpendicularly impinging on the wafer surface (along the $c$-axis of the GaN nanoLED crystal stack). The laser excitation energy $(2.38 \mathrm{eV})$ is below the bandgap energies of the InGaN/GaN MQWs $(2.79 \mathrm{eV})$ and $\mathrm{GaN}(3.39 \mathrm{eV})$, assuring that the Raman spectra purely originate in a nonresonance regime ${ }^{86}$. All three samples feature the $E_{2}$ (high) and $\mathrm{A}_{1}(\mathrm{LO})$ modes of hexagonal wurtzite $\mathrm{GaN}^{87}$. Transversal optical (TO) phonon modes (e.g., $\mathrm{A}_{1}$ (TO) 
mode) remain silent for the analysis of $\mathrm{GaN}$ in the $c$-axis orientation and thus are not obtained in the present backscattering configuration of the Raman setup ${ }^{86,88,89}$. In contrast, the $E_{2}$ (high) mode is very sensitive to strain and has been commonly employed to quantitatively determine the stress $(\sigma)$ in GaN epilayers ${ }^{90-92}$. Here, unstressed GaN is described with $E_{2}$ (high) at $567.2 \mathrm{~cm}^{-1}$ (dashed line in Fig. 6a), whereas compressive/tensile stress results in a shifted $\mathrm{E}_{2}$ (high) Raman peak ${ }^{88,91}$. The $\mathrm{E}_{2}$ (high) modes of the planar LED and the nanoLEDs before and after $f s$-LLO are detected at $568.4,566.6$, and $564.2 \mathrm{~cm}^{-1}$, respectively (see Fig. S5a in the Supplementary Information). Furthermore, the stress of each sample is estimated by using the following expression:

$$
\sigma=\frac{\Delta \omega}{k}
$$

with the difference between the measured and unstrained $\mathrm{E}_{2}$ (high) Raman modes $\left(\Delta \omega=567.2 \mathrm{~cm}^{-1}-\mathrm{E}_{2}\right.$ (high)) and a constant Raman stress coefficient $\left(k=4.3 \mathrm{~cm}^{-1}\right.$ $\left.\mathrm{GPa}^{-1}\right)^{91}$. Using Eq. (1), the planar $\mathrm{GaN}$ epilayer results in a compressive stress value of $\sigma=-0.279 \mathrm{GPa}$, whereas the nanoLED before $f s$-LLO reveals a nearly stress-free value of $\sigma=0.139 \mathrm{GPa}$. This result suggests that the originally compressively stressed InGaN/GaN LED epilayers are relaxed through transformation into nanowires by the hybrid etching (ICP-RIE and $\mathrm{KOH}$ etching) process with sufficiently high aspect ratios and free surface areas. After conducting $f$-LLO and subsequent transfer onto the foreign $\mathrm{Cu}$ substrate, the $\mathrm{E}_{2}$ (high) mode shifts even further towards lower wavenumbers and thus results in well-pronounced tensile stress of $\sigma=0.698 \mathrm{GPa}$. From other reports, such tensile stress normally occurred within the GaN LEDs that had been transferred from sapphire onto unconventional flexible substrates (e.g., polyimide and plastic $)^{92,93}$. For example, Horng et al. ${ }^{92}$ demonstrated that shifts of the $E_{2}$ (high) Raman mode are more pronounced for strongly bent samples. Furthermore, the GaN $A_{1}$ (TO) peak was observed at $533.2 \mathrm{~cm}^{-1}$ after the patterning of the nanoLED and the $f s$-LLO process (Fig. 6a, blue and green lines), which might be because during Raman measurement, the laser was also scattered by planes other than the $c$-plane on $\mathrm{GaN}$ nanowire surfaces $^{94,95}$.

X-ray diffraction (XRD) $2 \theta$ scans of the as-grown planar LED, on-wafer nanoLED, and $f s$-LLO-transferred nanoLED samples were employed to assess potential changes in the GaN crystal quality that occurred during the nanopatterning and transfer processes. In the X-ray diffractograms of nanoLEDs before and after $f s$-LLO, the (0002) main peak of GaN slightly shifted from $34.56^{\circ}$ to a lower diffraction angle to $34.54^{\circ}$, whereas the full width at half maximum values remained constant at $0.28^{\circ}$ (Fig. 6b).
This indicates that the GaN crystal did not degrade substantially after being transferred onto $\mathrm{Cu}$ foil ${ }^{96}$. However, the intensity ratio between the (0004) and (0002) peaks of $\mathrm{GaN}$ decreased, and their satellite peaks were reduced after $f s$-LLO (Fig. S5b in the Supplementary Information). A similar intensity ratio change was observed in $\mathrm{GaN}$ samples etched in sulfuric/phosphoric acid solutions ${ }^{97}$, in which samples with larger strains seem to have a smaller $(0004) /(0002)$ ratio. This suggests that the $f s$-LLO process introduces additional tensile strain, which agrees with the Raman spectroscopy results, as discussed above. Moreover, a peak of (00012) sapphire with a very low intensity was apparent, indicating that slight sapphire debris was attached to the released GaN during the transfer process (see Fig. S5c in the Supplementary Information).

PL spectra (excitation energy of $3.81 \mathrm{eV}$ ) for all three samples (i.e., as-grown unpatterned InGaN/GaN LED material, as-etched nanoLEDs, and as- $f s$-LLO-released nanoLEDs) are shown in Fig. 6c. The planar LED revealed a very pronounced PL peak at $\sim 445 \mathrm{~nm}$ attributed to the recombination of electron-hole pairs in the InGaN/GaN MQWs. In contrast, the PL emission of the GaN signal (at $\sim 363-366 \mathrm{~nm}$ ) was barely visible in the planar LED because most of the excitation laser was absorbed inside the upper regions of the LED stack. After nanowire structuring by ICP-RIE, the underlying $\mathrm{GaN}$ buffer layer was directly exposed to the PL laser beam. Consequently, this led to an intensified PL signal of the GaN peak at $\sim 363 \mathrm{~nm}$. Although the peak attributed to the MQW slightly decreased, which could be explained by a reduction in the active emission area inside the excitation volume, the PL peak wavelength remained at $445.1 \mathrm{~nm}$, suggesting no severe differences in the InGaN/GaN layers between the planar and nanoLEDs. However, more pronounced changes in the peak positions were found after $f s$-LLO. In particular, a broader PL signal at a wavelength of $\sim 489 \mathrm{~nm}$ was discovered that was ascribed to the underlying $\mathrm{Cu}$ substrate (Fig. S5d in the Supplementary Information). The nanoLED after $f s$-LLO also exhibited a redshift of the GaN peak to $\sim 366 \mathrm{~nm}$, which could be explained by an expansion of the crystal lattice that qualitatively agrees with the increase in tensile stress obtained from the Raman analysis (cf. Fig. 6a). Although this is a reasonably consistent finding, it should be noted that the spatial resolutions of the micro-PL and micro-Raman systems are not identical, which limits further quantitative correlations at this point. Finally, a blueshift of the InGaN/ $\mathrm{GaN}$ peak to $439.9 \mathrm{~nm}$ was observed, indicating a similar trend to that reported for planar structures after conventional LLO. This could be ascribed to segregation and subsequent reduction of In content in the $\mathrm{MQW}^{98}$ or a weakening of the piezoelectric field that originated from a relaxation of the compressive stress and a consequent 
band flattening through which the overlap area of the electron and hole wave functions increased (which is equivalent to weakening the quantum-confined Stark effect ${ }^{90}$.

Furthermore, to identify the crystal quality and to confirm the complete stacking structure of the released GaN nanoLED after the $f s$-LLO process, cross-sectional images were obtained using focus-ion beam milling combined with SEM, as displayed in Fig. 6d. Here, the GaN nanoLED was still supported by the $500 \mathrm{~nm}$ thick $n$-GaN film underneath. In addition, we also explored thin-film GaN LEDs processed by identical $f s$-LLO recipes for comparison purposes (see Fig. 6e). Although little laser-induced damage was revealed at the bottom part of $n-\mathrm{GaN}$, which had a direct interface with the sapphire substrate prior to $f_{s}$-LLO, the LED layer structure including the 16 -fold InGaN/GaN MQW layers was intact, and no cracks were found. The depth of these superficial damages caused by laser absorption was $\sim 1-2 \mu \mathrm{m}$ from the LLO interface (see Fig. 6e). This result is similar to those yielded in other reports from conventional LLO cases $^{99}$, in which the severity and location of structural defects or damage depend on several factors (e.g., applied laser pulse width, wavelength, and $\mathrm{GaN}$ absorption coefficient). From those studies, the frequency-tripled Nd:YAG laser $(355 \mathrm{~nm})$ and the $\mathrm{KrF}$ pulsed excimer laser $(248 \mathrm{~nm})$ possessed $\mathrm{GaN}$ absorption coefficient values of $6 \times 10^{4} \mathrm{~cm}^{-1}$ and $2 \times 10^{5} \mathrm{~cm}^{-1}$, respectively ${ }^{99,100}$. Furthermore, a femtosecond laser with an energy range of $0.80-2.25 \mathrm{eV}$ (wavelength of $1550-550 \mathrm{~nm}$ ) was reported to result in two-photon absorption coefficient values on $\mathrm{GaN}$ between $1 \times 10^{-11}$ and $2.9 \times 10^{-11} \mathrm{~m} / \mathrm{W}^{101}$. However, for our case, a more detailed study related to the $\mathrm{GaN}$ absorption coefficient and damage mechanisms caused by $f s$-LLO using a $520 \mathrm{~nm}$ wavelength still needs to be performed. In addition to the structural and optical characterizations discussed, the transferred nanoLEDs will have to be further investigated in terms of their final processing steps (e.g., $p$-contact creation) and optoelectrical measurements (e.g., cathodoluminescence tests) to better understand the $f s$-LLO mechanism and to further assess the potential of this technique as a versatile and fast method to realize large-scale GaN nanoLED-based flexible devices.

\section{Conclusions}

A scalable approach to fabricate and transfer top-down III-nitride nanoLEDs from sapphire onto unconventional substrates has been developed. This approach relies on hybrid etching (i.e., a combination of ICP-RIE and $\mathrm{KOH}$-based etching processes), fs-LLO, and thermal adhesive bonding. This method has been demonstrated using full two-inch $\mathrm{GaN}$ wafers with a transfer yield of up to $\sim 99.5 \%$ by maintaining the vertical alignment of the nanoLEDs. To optimize the process in view of future nanoLED production, the hybrid dry-wet etching time was well controlled. After being irradiated with ultrashort laser pulses, the released $\mathrm{GaN}$ bottom surface was treated in $\mathrm{HCl}$ cleaning solution to remove all the $\mathrm{Ga}$ debris resulting from laser irradiation and Ga surface oxide $\left(\mathrm{Ga}_{2} \mathrm{O}_{3}\right)$. Based on all the measured structural and optical characteristics of the released $\mathrm{GaN}$ nanoLEDs on $\mathrm{Cu}$ foil, we conclude that their performance and quality can be comparable, according to the measured parameters, with those of original as-grown planar LEDs on sapphire. The compressive stress can even be reduced after nanowire formation, resulting in more relaxed InGaN/GaN MQWs. Despite requiring further processing and characterization steps to realize a fully functioning LED device (i.e., creation of $p$-contact, electroluminescence test, and quantum efficiency analysis), $f_{s}$-LLO has shown promising results as an effective nanoLED transfer route for next-generation highthroughput flexible display assembly.

\section{Materials and methods InGaN/GaN LED wafers}

Planar InGaN/GaN blue LED wafers were acquired from the epitaxy competence center $\left(\mathrm{ec}^{2}\right)$ at Technische Universität Braunschweig, Germany and E-Wave Corporation, the United Kingdom (UK). The $f s$-LLO, AFM, Raman spectroscopy, XRD, PL, and AES measurements were performed with E-Wave LED wafers, and the other experimental results presented in this report were achieved with LED wafers from $\mathrm{ec}^{2}$. For the samples produced in $\mathrm{ec}^{2}$, the MOVPE method was used to grow the LED layers on a two-inch $c$-plane (0001) double-side polished (DSP) sapphire substrate with a thickness of $430 \mu \mathrm{m}$ inside an Aixtron G3 reactor. Transparent DSP substrates were required to enable the transmission of light from the $f_{s}$-laser during the lift-off process. The epitaxial LED layer stack with a total thickness of $\sim 5 \mu \mathrm{m}$ consisted of a $2.4 \mu \mathrm{m}$ thick $n-\mathrm{GaN}$ buffer layer with a dopant concentration of $9 \times 10^{18} \mathrm{~cm}^{-3}$, a $2.1 \mu \mathrm{m}$ thick $n$-GaN layer with a dopant concentration of $2 \times$ $10^{19} \mathrm{~cm}^{-3}$, a fourfold multiquantum well (MQW, $13.6 \mathrm{~nm}$ period) with $\mathrm{InGaN} / \mathrm{GaN}$ layers $(2.5-3.0 \mathrm{~nm}$ and $10.5-11.0 \mathrm{~nm})$, and a $120 \mathrm{~nm}$ thick $p$-GaN layer. Commercial two-inch epitaxial InGaN/GaN LED wafers with a center wavelength of $455 \mathrm{~nm}$ and DSP sapphire substrates were purchased from E-Wave Corporation, UK. From their provided data, the total thickness of the whole LED layer stack is $\sim 4 \mu \mathrm{m}$. A single LED wafer consists of a $3.4 \mu \mathrm{m}$ thick $n-\mathrm{GaN}$ layer with a dopant concentration of $\sim 1.5 \times 10^{19} \mathrm{~cm}^{-3}$, a $0.4 \mu \mathrm{m}$ thick active layer having 16 pairs of InGaN/GaN MQWs, and a $0.3 \mu$ m thick $p$-GaN layer with a dopant concentration of $\sim 8.9 \times 10^{18} \mathrm{~cm}^{-3}$. 


\section{Top-down GaN nanoLED fabrication}

A process combination of photolithography, ICP-RIE, and wet etching was employed to fabricate top-down $\mathrm{GaN}$ nanoLEDs. First, a planar GaN LED wafer was cleaned in a boiled mixture of $30 \% \mathrm{H}_{2} \mathrm{O}_{2}$ and $98 \% \mathrm{H}_{2} \mathrm{SO}_{4}$ (1:1) for $5 \mathrm{~min}$, followed by a dip in $6.5 \%$ buffered HF to eliminate undesired organic contaminants. An MJB4 mask aligner (SÜSS MicroTec SE, Germany) was used to perform optical lithography, resulting in circular $\mathrm{Cr}$ etch masks with a thickness of $300 \mathrm{~nm}$ and a diameter of $1 \mu \mathrm{m}$. Prior to the creation of those patterns, a $300 \mathrm{~nm}$ thick $\mathrm{Cr}$ layer was deposited by the electron beam evaporation method, which was then followed by a chemical lift-off process. The first physical etching (ICP-RIE) of GaN films by an SI $500 \mathrm{C}$ plasma dry etcher (SENTECH Instruments $\mathrm{GmbH}$, Germany) was conducted to define the height of vertically standing nanoLEDs. Here, a typical dry etching recipe was used for our structures (i.e., ICP power of $800 \mathrm{~W}$, HF power of $275 \mathrm{~W}$, pressure of $0.5 \mathrm{~Pa}, \mathrm{SF}_{6} / \mathrm{H}_{2}$ fluxes of $12 \mathrm{sccm}$ and $100 \mathrm{sccm}$ at room temperature). To smoothen the rough sidewall surfaces of the $\mathrm{GaN}$ nanowires and simultaneously reduce their diameters, the sample was etched using a $\mathrm{KOH}$-based wet-chemical etchant for $20 \mathrm{~min}$ at a temperature of $80^{\circ} \mathrm{C}$.

After the GaN nanoLED arrays had been prepared, they were spin-coated using SU-8 resist with a spin speed of $4000 \mathrm{rpm}$ for $35 \mathrm{~s}$ and subsequently heated at $95^{\circ} \mathrm{C}$ for $45 \mathrm{~s}$. The SU-8 coating membrane possessed a thickness of $\sim 5 \mu \mathrm{m}$ filling the gaps and covered the nanowires. After the nanowires had been fully covered by SU-8, ICP-RIE was carried out to etch back the embedded SU-8 layer on the sample for $1-2 \mathrm{~min}$ (i.e., ICP power of $1000 \mathrm{~W}, \mathrm{HF}$ power of $15 \mathrm{~W}, \mathrm{SF}_{6} / \mathrm{O}_{2}$ fluxes of $5 / 49.5 \mathrm{sccm}$, pressure of $0.4 \mathrm{~Pa}$, and temperature of $23^{\circ} \mathrm{C}$ ). Hence, the top surface of $p$-GaN could finally be freely exposed, while the MQW and $n$-GaN layers were still embedded in the SU-8 membrane. The GaN nanoLEDs on sapphire were then temporarily bonded onto a borosilicate glass carrier utilizing thermal glue of Crystalbond 509 (Plano GmbH, Germany) with a melting point at $120^{\circ} \mathrm{C}$. Afterwards, the layer stack was left at room temperature for at least $5 \mathrm{~min}$. Hence, the glue could be hardened, yielding sapphire/ GaN nanoLEDs on SU-8/Crystalbond glue/borosilicate glass layers. After releasing the nanoLEDs from the sapphire in the $f s$-LLO transfer process, GaN nanoLEDs on SU-8/Crystalbond glue/borosilicate glass films were attached onto a sticky $\mathrm{Cu}$ foil, where their bonding became stronger than that of Crystalbond adhesive at a temperature of $120^{\circ} \mathrm{C}$. Hence, the borosilicate glass could easily be separated (see Video S1 in the Supplementary Material). As a result, the GaN nanoLEDs in the SU-8 membrane were finally fixed onto the $\mathrm{Cu}$ foil, where a subsequent cleaning procedure with acetone and isopropanol was also conducted to remove the residues of
Crystalbond glue. To prove the functionality of the GaN nanoLED device on $\mathrm{Cu}$ foil after $f s$-LLO, an electroluminescence test was conducted using a semiconductor characterization system (4200-SCS Keithley, Keithley Instruments $\mathrm{GmbH}$ ), as shown in Fig. S6a in the Supplementary Information. It was obvious that blue light was emitted from the GaN nanoLED, although its emission was localized only in a certain area where the microneedle probe was applied on the $p$-GaN contact of the GaN nanoLED. It should be noted that in this study, we have not yet optimized both $p$ - and $n$-contacts (i.e., silver nanowires or ITOs were not used in this sample). The I-V characteristics of a single $f s$-LLO-processed GaN nanoLED during electroluminescence tests are depicted in Fig. S6b in the Supplementary Information.

\section{Laser micromachining setup}

In the $f s$-LLO transfer experiments, a Yb-based commercial $f s$-laser source (SPIRIT-1040, Newport SpectraPhysics GmbH, Germany) with a peak wavelength of $520 \mathrm{~nm}$, a pulse width of $350 \mathrm{fs}$, and a repetition rate of $200 \mathrm{kHz}$ was used. The emitted laser beam passed through a galvanometer scanner (IntelliSCAN III, SCANLAB GmbH, Germany) approaching a telecentric $f$-theta objective, which was then directed to a sample positioner. The mirrors inside the galvanometer scanner allowed fast $x-y$ beam scanning along the sample surface with a velocity of up to $3 \mathrm{~m} / \mathrm{s}$, where the incident angle was kept nearly orthogonal to the surface by the telecentric objective. A further detailed description of the $f s$-LLO setup can be found in our previous report ${ }^{35}$.

\section{Material characterization}

To investigate the crystal quality of GaN nanoLEDs before and after $f s$-LLO, XRD measurements were carried out using a Bruker D8 Advance with $\mathrm{Cu} K \alpha$ radiation $(\lambda=0.154 \mathrm{~nm})$, an acceleration voltage of $40 \mathrm{kV}$, current of $40 \mathrm{~mA}$, and Bragg-Brentano $(\theta-2 \theta)$ configuration. The PL characterization of GaN nanoLEDs was conducted under continuouswave excitation (Kimmon, $\mathrm{HeCd}$ laser, $\lambda_{\mathrm{ex}}=325 \mathrm{~nm}$ ). The PL was collected with an objective and imaged onto the entrance slit of a spectrometer. Raman spectra were captured on a Renishaw inVia Qontor Raman microscope equipped with an excitation wavelength of $532 \mathrm{~nm}$ and a $\times 100$ zoom lens. The GaN nanoLED cross-section was investigated using a Thermo Fisher Helios 5 UX Dual Beam microscope.

The AES spectra of GaN surfaces were recorded using an Omicron NanoSAM Auger electron microscope equipped with a Zeiss Supra field-emission scanning electron microscope (FE-SEM) and a hemispherical electron energy analyzer. The measurements were performed in constant retard ratio scan mode employing a $5 \mathrm{kV}$ electron beam for the excitation source with a tilt 
angle of $30^{\circ}$ to the sample normal position. The AES spectra were acquired on $4 \times 4 \mu^{2}$ selected areas. To observe the surface morphology and roughness of the fabricated GaN nanoLEDs, SEM (Leica Cambridge S360FE) and AFM in tapping mode (Veeco Dimension 3100) were employed. The AFM surface scans were measured on an area of $15 \times 15 \mu \mathrm{m}^{2}$ in tapping mode using a silicon microcantilever. All these characterizations were carried out at room temperature.

\section{Acknowledgements}

This work was funded in part by the Lower Saxony Ministry for Science and Culture (N-MWK) within the group of "LENA-OptoSense", in part by the European Union's Horizon 2020 research and innovation program within the project of "ChipScope-Overcoming the Limits of Diffraction with SuperResolution Lighting on a Chip" under grant agreement no. 737089, and in part by the Deutsche Forschungsgemeinschaft (DFG, German Research Foundation) within "Excellence Strategy of EXC-2123 QuantumFrontiers390837967". The authors thank Christoph Margenfeld, Irene Manglano Clavero, and Jana Hartmann for GaN LED wafer preparation in the $\mathrm{ec}^{2}$. Nursidik Yulianto, Nurhalis Majid, and Andam Deatama Refino acknowledge the Ministry of Research, Technology and Higher Education of the Republic of Indonesia (RISTEKDIKTI) for the Ph.D. scholarships of RISET-Pro under no. 34/ RISET-Pro/FGS/III/2019, RISET-Pro under no. 35/RISET-Pro/FGS/III/2019, and BPPLN under no. T/912/D3.2/KD.02.01/2019. Support from the Indonesian-German Centre for Nano and Quantum Technologies (IG-Nano) is also acknowledged. Alina Syring gratefully acknowledges support by the DFG Research Training Group GrK1952/1 "Metrology for Complex Nanosystems" and the "Braunschweig International Graduate School of Metrology (B-IGSM)". Florian Meierhofer and Tobias Voss also acknowledge financial support from the strategic research initiative "Quantum- and Nanometrology (QUANOMET)". The authors thank Angelika Schmidt, Juliane Breitfelder, Aileen Michalski, and Diana Herz for their technical support during the experiments and Steffen Bornemann for his assistance with the fs-laser micromachining setup development.

\section{Author details}

${ }^{1}$ Institute of Semiconductor Technology (IHT), Technische Universität Braunschweig, Hans-Sommer-Straße 66, Braunschweig 38106, Germany. ${ }^{2}$ Laboratory for Emerging Nanometrology (LENA), Technische Universität Braunschweig, Langer Kamp 6, Braunschweig 38106, Germany. ${ }^{3}$ Research Center for Physics, Indonesian Institute of Sciences (LIPI), JI. Kawasan Puspiptek No. 441-442, Tangerang, Selatan 15314, Indonesia. ${ }^{4}$ Engineering Physics Program, Institut Teknologi Sumatera (ITERA), J. Terusan Ryacudu, Way Huwi, Lampung Selatan, Lampung 35365, Indonesia. ${ }^{5}$ Institute of Energy Research and Physical Technologies, Technische Universität Clausthal, Leibnizstraße 4, Clausthal-Zellerfeld 38678, Germany. ${ }^{6}$ Institute for Solar Fuels, HelmholtzZentrum Berlin für Materialien und Energie GmbH, Hahn-Meitner-Platz 1, Berlin 14109, Germany. 7 Department of Engineering Physics, Institut Teknologi Sepuluh Nopember (ITS), I. Arif Rahman Hakim, ITS Campus Sukolilo, Surabaya 60111, Indonesia. ${ }^{8}$ Department of Physics, Faculty of Mathematics and Natural Sciences, Universitas Gadjah Mada, Sekip Utara PO Box BLS 21, Yogyakarta 55281, Indonesia

\section{Author contributions}

H.S.W. and N.Y. conceived the idea and concept, designed the experiments, formulated the materials, validated the methods, interpreted the experimental results, created the figures, wrote the initial manuscript, and revised the paper. N.Y. fabricated the GaN nanoLEDs, performed laser lift-off processing, and measured contact angles. A.D.R. and S.M. carried out ICP-RIE and wet-chemical etching processes. A.S., F.M., and T.V. conducted PL characterization and Raman spectroscopy and analyzed their results. P.S. and F.F.A. performed XRD measurements and analyzed their results. N.M. and W.D. characterized the sample surface roughness and elemental composition using AFM and AES, respectively. R.A.W., K.T., and W.D. provided inputs on the analysis of material characteristics and revised the paper. H.S.W. and A.W. supervised the work, led the project, and acquired the funding. All authors discussed the results and approved the final manuscript.
Funding

Open Access funding enabled and organized by Projekt DEAL.

\section{Data availability}

All data generated or analyzed during this study are included in this published article (and its Supplementary Information and Material files).

\section{Conflict of interest}

The authors declare no competing interests.

Supplementary information The online version contains supplementary material available at https://doi.org/10.1038/s41378-021-00257-y.

Received: 7 January 2021 Revised: 24 February 2021 Accepted: 24 February 2021

Published online: 23 April 2021

\section{References}

1. Nakamura, S. \& Krames, M. R. History of gallium-nitride-based light-emitting diodes for illumination. Proc. IEEE 101, 2211-2220 (2013).

2. Nakamura, S. Nobel Lecture: Background story of the invention of efficient blue InGaN light emitting diodes. Rev. Mod. Phys. 87, 1139-1151 (2015).

3. Wasisto, H. S., Prades, J. D., Gülink, J. \& Waag, A. Beyond solid-state lighting: miniaturization, hybrid integration, and applications of GaN nano-and microLEDs. Appl. Phys. Rev. 6, 041315 (2019).

4. Markiewicz, N. et al. Micro light plates for low-power photoactivated (gas) sensors. Appl. Phys. Lett. 114, 053508 (2019).

5. Goßler, C. et al. GaN-based micro-LED arrays on flexible substrates for optical cochlear implants. J. Phys. D. Appl. Phys. 47, 205401 (2014).

6. Scholz, G. et al. Continuous live-cell culture imaging and single-cell tracking by computational lensfree LED microscopy. Sensors 19, 1-13 (2019).

7. Schmidt, I. et al. Gold-modified indium tin oxide as a transparent window in optoelectronic diagnostics of electrochemically active biofilms. Biosens. Bioelectron. 94, 74-80 (2017).

8. Casals, O. et al. A parts per billion (ppb) sensor for $\mathrm{NO}_{2}$ with microwatt $(\mu \mathrm{W})$ power requirements based on micro light plates. ACS. Sensors 4, 822-826 (2019).

9. Caccamo, L. et al. Band engineered epitaxial 3D GaN-InGaN core-shell rod arrays as an advanced photoanode for visible-light-driven water splitting. ACS Appl. Mater. Interfaces 6, 2235-2240 (2014).

10. Lin, J. Y. \& Jiang, H. X. Development of microLED. Appl. Phys. Lett. 116, 100502 (2020).

11. Liu, X. et al. Submicron full-color LED pixels for microdisplays and micro-LED main displays. J. Soc. Inf. Disp. 28, 410-417 (2020).

12. Bian, J., Zhou, L., Yang, B., Yin, Z. \& Huang, Y. A. Theoretical and experimental studies of laser lift-off of nonwrinkled ultrathin polyimide film for flexible electronics. Appl. Surf. Sci. 499, 143910 (2020).

13. Park, J. B. et al. Transfer printing of vertical-type microscale light-emitting diode array onto flexible substrate using biomimetic stamp. Opt. Express 27 6832 (2019).

14. Li, S.-H. et al. Performance analysis of GaN-based micro light-emitting diodes by laser lift-off process. Solid State. Electron. Lett. 1, 58-63 (2019).

15. Park, J-B. et al. Stable and efficient transfer-printing including repair using a GaN-based microscale light-emitting diode array for deformable displays. Sci. Rep. 9, 11551 (2019)

16. Kim, J., Kim, J. H., Cho, S. H. \& Whang, K. H. Selective lift-off of GaN lightemitting diode from a sapphire substrate using 266-nm diode-pumped solid-state laser irradiation. Appl. Phys. A Mater. Sci. Process. 122, 1-6 (2016).

17. Robin, $Y$. et al. Insight into the performance of multi-color InGaN/GaN nanorod light emitting diodes. Sci. Rep. 8, 7311 (2018).

18. Nami, M. et al. Carrier dynamics and electro-optical characterization of highperformance GaN/InGaN core-shell nanowire light-emitting diodes. Sci. Rep. 8, 501 (2018).

19. Asad, M. et al. Optically invariant InGaN nanowire light-emitting diodes on flexible substrates under mechanical manipulation. npj Flex. Electron 3, 1-6 (2019). 
20. Li, S. \& Waag, A. GaN based nanorods for solid state lighting. J. Appl. Phys. 111, 205401 (2012)

21. Fernández-Garrido, S. et al. Top-down fabrication of ordered arrays of GaN nanowires by selective area sublimation. Nanoscale Adv. 1, 1893-1900 (2019).

22. Yoshizawa, M., Kikuchi, A., Mori, M., Fujita, N. \& Kishino, K. Growth of selforganized GaN nanostructures on Al2O3(0001) by RF-radical source molecular beam epitaxy. Jpn. J. Appl. Phys. 36, L459-L462 (1997).

23. Barrigón, E., Heurlin, M., Bi, Z., Monemar, B. \& Samuelson, L. Synthesis and applications of III-V nanowires. Chem. Rev. 119, 9170-9220 (2019).

24. Li, Q. et al. Optical performance of top-down fabricated InGaN/GaN nanorod light emitting diode arrays. Opt. Express 19, 25528 (2011).

25. Zhang, S. et al. On the efficiency droop of top-down etched InGaN/GaN nanorod light emitting diodes under optical pumping. AIP Adv. 3, 082103 (2013).

26. Mariana, S. et al. Vertical GaN nanowires and nanoscale light-emitting-diode arrays for lighting and sensing applications. ACS Appl. Nano Mater. 2, 4133-4142 (2019).

27. Dai, X. et al. Flexible light-emitting diodes based on vertical nitride nanowires. Nano Lett. 15, 6958-6964 (2015).

28. Guan, N. et al. Flexible white light emitting diodes based on nitride nanowires and nanophosphors. ACS Photonics 3, 597-603 (2016).

29. Guan, N. et al. Colour optimization of phosphor-converted flexible nitride nanowire white light emitting diodes. J. Phys. Photonics 1, 035003 (2019).

30. Kapoor, A. et al. Green electroluminescence from radial $m$-Plane InGaN quantum wells grown on gan wire sidewalls by metal-organic vapor phase epitaxy. ACS Photonics 5, 4330-4337 (2018).

31. Chung, K, Lee, C.-H. \& Yi, G.C. Transferable GaN layers grown on ZnO-coated graphene layers for optoelectronic devices. Science 330, 655-657 (2010).

32. $\mathrm{Ha}$, J. et al. The fabrication of vertical light-emitting diodes using chemical liftoff process. IEEE Photonics Technol. Lett. 20, 2007-2009 (2008).

33. Kobayashi, Y., Kumakura, K, Akasaka, T. \& Makimoto, T. Layered boron nitride as a release layer for mechanical transfer of GaN-based devices. Nature $\mathbf{4 8 4}$ 223-227 (2012).

34. Hwang, D. et al. Photoelectrochemical liftoff of LEDs grown on freestanding c-plane GaN substrates. Opt. Express 24, 22875-22880 (2016).

35. Bornemann, S. et al. Femtosecond laser lift-off with sub-bandgap excitation for production of free-standing GaN light-emitting diode chips. Adv. Eng. Mater. 22, 1901192, (2019).

36. Yulianto, N. et al. Ultrashort pulse laser lift-off processing of InGaN/GaN lightemitting diode chips. MACS Appl. Electron Mater 3, 778-788 (2021).

37. Voronenkov, V. et al. Laser slicing: a thin film lift-off method for GaN-on-GaN technology. Results Phys. 13, 27-30 (2019).

38. Tian, Z. et al. Super flexible GaN light emitting diodes using microscale pyramid arrays through laser lift-off and dual transfer. Opt. Express 26, 1817 (2018).

39. Tian, X., Chen, W. \& Zhang, J. Thermal design for the high-power LED lamp. J. semicond. 32, 1-4 (2011).

40. Schmidt, M. P. et al. Flexible free-standing SU-8 microfluidic impedance spectroscopy sensor for 3-D molded interconnect devices application. J. Sens. Sens. Syst. 1, 55-61 (2016).

41. Spratley, J. P. F., Ward, M. C. L. \& Hall, P. S. Highly flexible SU-8 microstructures. Solid-State Sensors, Actuators Microsystems Conference IEEE. 587-590 (2007).

42. Wasisto, H. S. et al. Silicon resonant nanopillar sensors for airborne titanium dioxide engineered nanoparticle mass detection. Sens. Actuators, B Chem. 189, 146-156 (2013).

43. Merzsch, S. et al. Production of vertical nanowire resonators by cryogenicICP-DRIE. Microsyst. Technol. 20, 759-767 (2014).

44. Hamdana, G. et al. Towards fabrication of 3D isotopically modulated vertical silicon nanowires in selective areas by nanosphere lithography. Microelectron. Eng. 179, 74-82 (2017).

45. Wasisto, H. S., Merzsch, S., Steib, F., Waag, A. \& Peiner, E. Vertical silicon nanowire array-patterned microcantilever resonators for enhanced detection of cigarette smoke aerosols. Micro Nano Lett. 9, 676-679 (2014).

46. Fatahilah, M. F. et al. Traceable nanomechanical metrology of GaN micropillar array. Adv. Eng. Mater. 20, 1800353 (2018).

47. Yonenaga, I., Hoshi, T. \& Usui, A. Hardness of bulk single-crystal gallium nitride at high temperatures. Jpn. J. Appl. Phys., Part 2 Lett. 39, 12-14 (2000).

48. Awan, K. M. et al. Fabrication and optical characterization of GaN waveguides on (-201)-oriented $\beta$-Ga2O3. Opt. Mater. Express 8, 88 (2018).
49. Chang, L. B., Liu, S. S. \& Jeng, M. J. Etching selectivity and surface profile of $\mathrm{GaN}$ in the $\mathrm{Ni}, \mathrm{SiO} 2$ and photoresist masks using an inductively coupled plasma. Jpn. J. Appl. Phys. 40, 1242-1243 (2001).

50. Wang, G. T., Li, Q., Wierer, J. J., Koleske, D. D. \& Figiel, J. J. Top-down fabrication and characterization of axial and radial II-nitride nanowire LEDs. Phys. Status Solidi. Appl. Mater. Sci. 211, 748-751 (2014).

51. Yu, F. et al. GaN nanowire arrays with nonpolar sidewalls for vertically integrated field-effect transistors. Nanotechnology 28, 095206 (2017).

52. Steglich, M. et al. The structural and optical properties of black silicon by inductively coupled plasma reactive ion etching. J. Appl. Phys. 116, 173503 (2014).

53. Liu, X. et al. Black silicon: fabrication methods, properties and solar energy applications. Energy Environ. Sci. 7, 3223-3263 (2014).

54. Pearton, S. J., Shul, R. J. \& Ren, F. A review of dry etching of GaN and related materials. MRS Internet J. Nitride Semicond. Res. 5, 1-38 (2000).

55. Okada, N. et al. Formation of distinctive structures of GaN by inductivelycoupled-plasma and reactive ion etching under optimized chemical etching conditions. AIP Adv. 7, 065111 (2017).

56. Fatahilah, M. F. et al. 3D GaN nanoarchitecture for field-effect transistors Micro Nano Eng. 3, 59-81 (2019).

57. Fatahilah, M. F. et al. Top-down GaN nanowire transistors with nearly zero gate hysteresis for parallel vertical electronics. Sci. Rep. 9, 10301 (2019).

58. Yu, F. et al. Vertical architecture for enhancement mode power transistors based on GaN nanowires. Appl. Phys. Lett. 108, 213503 (2016).

59. Paramanik, D. et al. Fabrication of high quality GaN nanopillar arrays by dry and wet chemical etching. arXiv Prepr. 1311, 0321 (2013).

60. Zhou, S., Cao, B. \& Liu, S. Optimized ICP etching process for fabrication of oblique GaN sidewall and its application in LED. Appl. Phys. A Mater. Sci. Process. 105, 369-377 (2011).

61. Vartuli, C. B. et al. ICP dry etching of III-V nitrides. Materials Research Society Symposium - Proceedings, 468 (1997).

62. Wong, J. C. et al. Selective anisotropic etching of GaN over AlGaN for very thin films. J. Vac. Sci. Technol. A Vac., Surf., Film. 36, 030603 (2018).

63. Zhou, S., Cao, B. \& Liu, S. Dry etching characteristics of GaN using $\mathrm{Cl} 2 / \mathrm{BCl} 3$ inductively coupled plasmas. Appl. Surf. Sci. 257, 905-910 (2010).

64. Sreenidhi, T., Baskar, K, Dasgupta, A. \& Dasgupta, N. Reactive ion etching of GaN in SF6 + Ar and SF6 + N2 plasma. semicond. Sci. Technol. 23, 125019 (2008)

65. Olivier, F., Daami, A., Licitra, C. \& Templier, F. Shockley-Read-Hall and Auger non-radiative recombination in GaN based LEDs: a size effect study. Appl. Phys. Lett. 111, 022104 (2017).

66. Foster, C. M., Collazo, R., Sitar, Z. \& Ivanisevic, A. Aqueous stability of Ga- and $\mathrm{N}$-polar gallium nitride. Langmuir 29, 216-220 (2013).

67. Ng, H. M., Weimann, N. G. \& Chowdhury, A. GaN nanotip pyramids formed by anisotropic etching. J. Appl. Phys. 94, 650-653 (2003).

68. Tautz, M. \& Díaz Díaz, D. Wet-chemical etching of gan: underlying mechanism of a key step in blue and white LED production. ChemistrySelect 3, 1480-1494 (2018)

69. Tautz, M., Weimar, A., Graßl, C., Welzel, M. \& Díaz Díaz, D. Anisotropy and mechanistic elucidation of wet-chemical gallium nitride etching at the atomic level. Phys. Status Solidi Appl. Mater. Sci. 217, 2000221, (2020).

70. Chen, W. et al. GaN nanowire fabricated by selective wet-etching of GaN micro truncated-pyramid. J. Cryst. Growth 426, 168-172 (2015).

71. Maier, K., Helwig, A., Müller, G., Schörmann, J. \& Eickhoff, M. Photoluminescence detection of surface oxidation processes on InGaN/GaN nanowire arrays. ACS Sensors 3, 2254-2260 (2018).

72. Babichev, A. V. et al. GaN nanowire ultraviolet photodetector with a graphene transparent contact. Appl. Phys. Lett. 103, 201103-201103-4 (2013).

73. Jeong, G. J. et al. Silver nanowires for transparent conductive electrode to GaN-based light-emitting diodes. Appl. Phys. Lett. 106, 031118 (2015).

74. Huang, Y. et al. Highly transparent light emitting diodes on graphene encapsulated Cu nanowires network. Sci. Rep. 8, 1-11 (2018).

75. Oh, M. et al. Silver nanowire transparent conductive electrodes for highefficiency III-nitride light-emitting diodes. Sci. Rep. 5, 1-11 (2015).

76. Rasmussen, K. H., Keller, S. S., Jensen, F., Jorgensen, A. M. \& Hansen, O. SU-8 etching in inductively coupled oxygen plasma. Microelectron. Eng. 112, 35-40 (2013).

77. Reddy, J. W. et al. High density, double-sided, flexible optoelectronic neural probes with embedded $\mu \mathrm{LEDS}$. Front. Genet. 10, 1-15 (2019). 
78. Nakashima, S., Sugioka, K. \& Midorikawa, K. Fabrication of microchannels in single-crystal GaN by wet-chemical-assisted femtosecond-laser ablation. Appl. Surf. Sci. 255, 9770-9774 (2009).

79. He, R. et al. Micro-structure changes induced by femtosecond laser on the surface of GaN multilayer film grown on Si substrate. Appl. Phys. A Mater. Sci. Process. 123, 1-8 (2017).

80. King, S. W. et al. Cleaning of AIN and GaN surfaces. J. Appl. Phys. 84, 5248-5260 (1998).

81. Okada, $\mathrm{H}$. et al. Investigation of HCl-based surface treatment for GaN devices. AIP Conf. Proc. 1709, 020011 (2016).

82. Lee, C., Sekiguchi, H., Okada, H. \& Wakahara, A. Plasma-induced damage and recovery on $\mathrm{Au} / \mathrm{n}-\mathrm{GaN}$ Schottky diode in different processes. Jpn. J. Appl. Phys. 51, 076503 (2012).

83. Diale, M., Auret, F. D., Van Der Berg, N. G., Odendaal, R. Q. \& Roos, W. D. Analysis of GaN cleaning procedures. Appl. Surf. Sci. 246, 1-3 (2005).

84. Hossain, T. et al. Effect of GaN surface treatment on Al2O3/n-GaN MOS capacitors. J. Vac. Sci. Technol. B, Nanotechnol. Microelectron. Mater. Process. Meas. Phenom. 33, 061201 (2015).

85. Douglass, K., Hunt, S., Teplyakov, A. \& Opila, R. L. Surface cleaning procedures for thin films of indium gallium nitride grown on sapphire. Appl. Surf. Sci. $\mathbf{2 5 7}$ 1469-1472 (2010).

86. Sarau, G., Heilmann, M., Latzel, M. \& Christiansen, S. Disentangling the effects of nanoscale structural variations on the light emission wavelength of single nano-emitters: InGaN/GaN multiquantum well nano-LEDs for a case study. Nanoscale 6, 11953-11962 (2014).

87. Harima, H. Properties of GaN and related compounds studied by means of Raman scattering. J. Phys. Condens. Matter 14, R967 (2002).

88. Kuball, M. Raman spectroscopy of GaN, AlGaN and AIN for process and growth monitoring/control. Surf. Interface Anal. 31, 987-999 (2001).

89. Gao, H. et al. First and second order Raman scattering spectroscopy of nonpolar a -plane GaN. J. Appl. Phys. 101, 1-6 (2007).

90. Lei, Y. et al. Optical characterization of GaN-based vertical blue light-emitting diodes on P-type silicon substrate. Crystals 10, 1-12 (2020).
91. Park, B. G. et al. Comparison of stress states in GaN films grown on different substrates: langasite, sapphire and silicon. J. Cryst. Growth 425, 149-153 (2015)

92. Horng, R.-H., Tien, C.-H., Chuang, S.-H., Liu, K.-C. \& Wuu, D.-S. External stress effects on the optical and electrical properties of flexible InGaN-based green light-emitting diodes. Opt. Express 23, 31334 (2015).

93. Seo, J. H. et al. A simplified method of making flexible blue LEDs on a plastic substrate. IEEE Photonics J. 7, 6900108 (2015).

94. Chen, W. H. et al. Shock-assisted superficial hexagonal-to-cubic phase transition in GaN/Sapphire interface induced by using ultra-violet laser lift-of techniques. Chinese Phys. Lett. 26, 016203 (2009).

95. Hsiao, C. L. et al. Micro-Raman spectroscopy of a single freestanding GaN nanorod grown by molecular beam epitaxy. Appl. Phys. Lett. 90, 043102 (2007).

96. Lee, K. T. et al. Mechanism underlying damage induced in gallium nitride epilayer during laser lift-off process. Jpn. J. Appl. Phys. 47, 930-932 (2008)

97. Liu, J. et al. Investigation of cracks in gan films grown by combined hydride and metal organic vaporphase epitaxial method. Nanoscale Res. Lett. 6, 1-8 (2011).

98. Doan, M. H. et al. Influence of laser lift-off on optical and structural properties of InGaN/GaN vertical blue light emitting diodes. AIP Adv. 2, 022122 (2012)

99. Cheng, J.-H., Wu, Y. S., Peng, W. C. \& Ouyang, H. Effects of laser sources on damage mechanisms and reverse-bias leakages of laser lift-off GaN-based LEDs. J. Electrochem. Soc. 156, H640 (2009).

100. Wu, Y. S., Cheng, J. H., Peng, W. C. \& Ouyang, H. Effects of laser sources on the reverse-bias leakages of laser lift-off GaN-based light-emitting diodes. Appl. Phys. Lett. 90, 251110 (2007).

101. Almeida, G. F. B. et al. Third-order nonlinear spectrum of GaN under femtosecond-pulse excitation from the visible to the near infrared. Photonics 6, 69 (2019). 